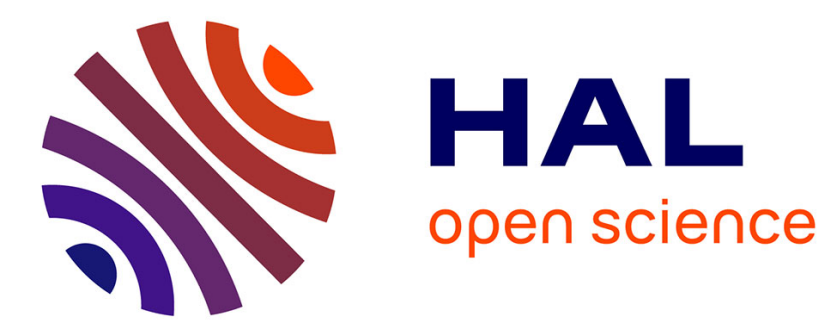

\title{
Spectral and modal energy transfer analyses of LES using the discontinuous Galerkin method and their application to the Variational Multiscale approach
}

Fabio Naddei, Marta de La Llave Plata, Eric Lamballais

\section{- To cite this version:}

Fabio Naddei, Marta de La Llave Plata, Eric Lamballais. Spectral and modal energy transfer analyses of LES using the discontinuous Galerkin method and their application to the Variational Multiscale approach. Journal of Computational Physics, 2021, 427, pp.110031. 10.1016/j.jcp.2020.110031 . hal03134563

\author{
HAL Id: hal-03134563 \\ https://hal.science/hal-03134563
}

Submitted on 8 Feb 2021

HAL is a multi-disciplinary open access archive for the deposit and dissemination of scientific research documents, whether they are published or not. The documents may come from teaching and research institutions in France or abroad, or from public or private research centers.
L'archive ouverte pluridisciplinaire HAL, est destinée au dépôt et à la diffusion de documents scientifiques de niveau recherche, publiés ou non, émanant des établissements d'enseignement et de recherche français ou étrangers, des laboratoires publics ou privés. 


\title{
Spectral and modal energy transfer analyses of LES using the discontinuous Galerkin method and their application to the Variational Multiscale approach
}

\author{
Fabio Naddei ${ }^{\mathrm{a}}$, Marta de la Llave Plata ${ }^{\mathrm{a}}$, Eric Lamballais ${ }^{\mathrm{b}}$ \\ ${ }^{a}$ Department of Aerodynamics, Aeroelasticity and Acoustics, ONERA, France \\ ${ }^{b}$ Institute PPRIME, Université de Poitiers, France
}

\begin{abstract}
7 Abstract
In this work we perform a-priori analyses of the Discontinuous Galerkin (DG) Variational Multiscale (VMS) method for Large Eddy Simulation (LES). An analytical framework is introduced to study the ideal energy transfer between resolved and unresolved scales. The proposed framework is consistent with the discretization employed for the DG-LES simulations. The concept of modal eddy viscosity is also introduced which can be employed for the a-priori analysis of the DG-VMS method or spectral vanishing viscosity approaches. The developed framework is then applied to the analysis of the energy transfer in DG-LES by employing a DNS database of the Taylor-Green Vortex (TGV) at $\mathrm{Re}=5000,20000$ and 40000 . A-priori analyses are carried out for the Vreman [1] and all-all [2] variants of the DG-VMS approach. The performed analysis demonstrates that when the DG-LES resolution limit falls at the beginning of the dissipation range the assumption of large scales free of interaction with the unresolved scales is valid and the DG-VMS approach can replicate the ideal SGS dissipation spectrum. For coarser resolutions, typical of LES at high Reynolds numbers, the DG-VMS approach is unable to replicate the ideal energy transfer mechanism at the large-resolved scales. It is shown, a-priori, that a more accurate agreement can be obtained by employing a mixed Smagorinsky and DG-VMS approach with a fixed value of the scale-fraction parameter.
\end{abstract}

Keywords: High-order, discontinuous Galerkin, Variational Multiscale, a-priori analysis, spectral energy transfer

\section{Introduction}

Large Eddy Simulation (LES) is a well established methodology for the prediction of turbulent flows for applications ranging from fundamental research to industrial design [3, 4, 5]. The working principle of LES is to resolve only the large-scale turbulent eddies and model the effect of the unresolved scales by means of a subgrid-scale (SGS) closure, thereby reducing the computational cost of simulations with respect to Direct Numerical Simulation (DNS).

One of the fundamental traits of turbulence, that must be correctly reproduced by SGS models, is the physical mechanism of energy transfer between the resolved turbulent scales and the

URL: fabio.naddei@onera.fr (Fabio Naddei) 
unresolved scales. This energy transfer mechanism can be studied by analysing the non-linear interaction of the full velocity field such as that obtained from DNS or by theoretical analyses by employing an analytical energy spectrum.

Early theoretical analyses of the energy transfer mechanisms in turbulent flows were performed by Heisenberg [6] and Kraichnan [7]. These studies involved the choice of an analytical energy spectrum and an arbitrary convolution filter to separate resolved and unresolved scales and evaluate the corresponding non linear interactions and energy transfer. The energy transfer mechanism was represented by means of an additional spectral eddy viscosity acting on the resolved modes. Kraichnan [7] employed a sharp cut-off filter in Fourier space to separate resolved and unresolved scales in theoretical turbulence characterized by an infinite inertial range (i. e. assuming infinite Reynolds number). Under these conditions, Kraichnan identified the presence of a cusp in the spectral eddy viscosity, near the cut-off, and a plateau at lower wavenumbers, which is a manifestation of the significant interaction between the unresolved scales and the large resolved scales away from the cut-off.

A-priori numerical analyses were carried out by Domaradzki et al. [8] based on DNS of the Taylor-Green Vortex (TGV) flow at $\mathrm{Re}=3000$ by applying a sharp spectral filter to define the ideal LES solution. This work confirmed the presence of the cusp of the spectral eddy viscosity near the cut-off. However, differently from the studies of Kraichnan, a negligible energy transfer was observed at relatively lower wavenumbers. A similar result was observed by McComb and Young [9] who analysed the spectral eddy viscosity for homogeneous isotropic turbulence at microscale Reynolds number $\operatorname{Re}_{\lambda}=190$. In their work a plateau in the eddy viscosity was observed only for the coarsest resolution, indicating a negligible interaction between large resolved and unresolved scales. In contrast, Métais and Lesieur [10] identified a plateau in the spectral eddy viscosity evaluated from an LES of homogeneous isotropic turbulence at infinite Reynolds number.

Using a-priori testing, the ideal energy transfer and the effective eddy viscosity obtained by applying the LES filter to DNS data can be employed to evaluate SGS models and aid in their improvement. As an example, the Smagorinsky model [11], still widely employed due to its simplicity and robustness, has been shown in a-priori analyses to be overdissipative on the large-scale structures, confirming the observations from a-posteriori tests [12]. Using the same approach, it has been shown that even though its dynamic variant by Germano et al. [13] is able to provide the correct global SGS dissipation and near-wall scaling, it fails to reproduce the ideal energy transfer spectrum (also called SGS dissipation spectrum). Indeed, it introduces an insufficient amount of dissipation at high wavenumbers while exhibiting an overdissipative behaviour at low wavenumbers as shown $e . g$. by Hughes et al. [14].

A number of new SGS models have been developed with the aim of reducing these shortcomings of traditional LES techniques. One such model is the Variational Multiscale (VMS) approach proposed by Hughes et al. [15]. The VMS approach advocates the strict separation of the resolved velocity field into a large-scale component, containing the largest coherent structures of the flow, and a small-scale component by means of a high-pass projection filter. The model then relies on the spectral gap assumption such that the large resolved scales are assumed to be virtually free of SGS dissipation. Thus the model (e. g. the Smagorinsky model or its dynamic version) only acts on the small-scale resolved component of the velocity field. This approach therefore mimics the ideal energy transfer mechanism as described by Domaradzki et al. [8]. The VMS approach, originally developed in the context of stabilized finite element (FE) and spectral methods, has demonstrated very accurate results in the simulation of several turbulent flow configurations and has since been extended to finite volume (FV) and spectral element type 
methods such as the discontinuous Galerkin (DG) method. We refer to the reviews of Gravemeier [16], Ahmed et al. [17] and Rasthofer and Gravemeier [18] for an overview of the VMS approach and several variants that have been proposed by other authors.

The combination of the VMS approach with DG methods in particular presents several properties which are of great interest for the improvement of the quality and efficiency of LES [19] The DG methods have rapidly gained popularity for scale-resolving simulations due to their excellent scalability and their ability to achieve high-order accuracy on general meshes [20]. The variational framework on which these methods rely allows for the local separation of scales using polynomial basis functions, which can be employed for multi-level methods and the VMS approach. In contrast to the spectral method, the DG framework allows for the efficient separation of scales even while working on completely unstructured meshes without requiring complex spatial filters. Moreover, the high-order polynomial representation of the solution allows for a higher flexibility in the decomposition into large and small scale components, as compared to FV and low order FE methods. Finally, the use of discontinuous solution spaces allows for the straightforward local adaptation of the scale-separation operator.

There are however still several open questions which require specific analysis in the context of the DG-VMS method. These are: the effect of the LES filter on the effective eddy viscosity, the effect of the scale-separation operator and the calibration of the coefficient involved in the SGS model. While these questions are still the subject of current research in the context of the DG-VMS approach, several studies have been already carried out in the context of standard LES approaches based on convolution filters. The main conclusions of these works are briefly outlined below.

The effect of the LES filter. Leslie and Quarini [21] performed theoretical analyses by considering an infinite inertial range and a Gaussian filter. Their results demonstrated that, in contrast to what is obtained for a sharp spectral filter, the use of a Gaussian filter leads to a spectral eddy viscosity characterized by a plateau from low to high wavenumbers and a sharp decay as the wavenumber approaches $1 / \Delta$ ( $\Delta$ being the filter width). Moreover, in the case of a productiontype spectrum, the shape of the ideal eddy viscosity strongly depends on the ratio between the LES cut-off wavenumber $k_{c}$ and that corresponding to the energy production phenomena. Similar conclusions can be drawn from the work of Cerutti et al. [22] who evaluated the eddy viscosity from experimental measurements corresponding to the use of a mixed filter (spectral cut-off in one direction and top-hat filter in the other two directions). The outcome of this study led the authors to conclude that the use of a mixed viscosity-hyperviscosity model can improve the accuracy of LES simulations. More recently Lamballais et al. [23] have evaluated the eddy viscosity from the DNS of the TGV configuration at $\mathrm{Re}=20000$ and observed the presence of the plateau described by Kraichnan [7] employing a spectral cut-off filter.

It appears, therefore, that the validity of the spectral gap assumption needs to be carefully analysed depending on the LES filter employed in the simulation. The extension of these analyses to the case of the DG-VMS approach presents additional complications as the DG-projection filter is not a convolution filter (as explained in Sec. 2). Thus this topic deserves special attention.

The effect of the high-pass filter. The second open question is the effect of the high-pass filter on the quality of the VMS model. As regards the choice of the cut-off wavenumber $\bar{k}$ associated with the high-pass filter, in actual simulations, this parameter is often selected heuristically or by trying to match reference results. In early numerical experiments, Hughes et al. [15] and Hughes et al. [24] have used a high-pass spectral filter with $\bar{k}$ corresponding to a scale-fraction parameter 
$\beta=\bar{k} / k_{c}$ equal to 0.59 and 0.5 , respectively, when employing the VMS approach with constant model coefficient. In later works Hughes et al. [14] employed a scale-fraction parameter $\beta=0.5$ for the VMS approach based on the dynamic Germano procedure for the determination of the model constant. Holmen et al. [25] carried out a sensitivity analysis for the LES of the turbulent channel flow. The authors showed that the use of the dynamic procedure reduces the sensitivity of the VMS-LES to the scale-fraction parameter and optimal results were obtained for $\beta \approx 0.5$. In contrast, for the static variants optimal results were obtained for $\beta \approx 0.7$ and the quality of the solution quickly deteriorated for other values. Ramakrishnan and Collis [26] have shown that the optimal scale-fraction parameter might depend on the flow considered and resolution available. It was observed that the optimal high-pass filter length can be related to the characteristic length of coherent structures of the flow. However they remarked that the small-scale space should contain at least $50 \%-60 \%$ of all modes to provide high quality first and second-order statistics.

The effect of the type of the high-pass filter in VMS was also analysed by Sagaut and Levasseur [27] and Meyers and Sagaut [28]. It was observed that the sharp cut-off filter (orthogonal in Fourier space) can provide an overdissipative behaviour at high wavenumbers leading to a bottleneck effect and the generation of a middle-wavenumber pile-up. Moreover, a discrete jump might appear in the energy spectrum near the high-pass filter cut-off (as observed by Meyers and Sagaut [28]). The use of a non-orthogonal high-pass filter (e. g. Gaussian filter) led to improved results by rendering all scales sensitive to the subgrid closure. Similar results were reported by Meyers and Sagaut [28] who further noted a reduced dependency on $\beta$ when employing a Gaussian filter. Other non-orthogonal high-pass filters have been employed in the Regularized Variational Multiscale (RVM) model by Jeanmart and Winckelmans [12]. The regular filter used by this model is obtained by iterating a tensor product compact discrete filter which can be efficiently applied in physical space for Finite Difference methods.

Calibration of the SGS model constant. As regards the value of the constant involved in the VMS model, a calibration has been derived by Hughes et al. [15] using the procedure due to Lilly [29]. The procedure assumes an infinite Reynolds number (infinite inertial range) and the calibration was obtained considering an isotropic sharp spectral filter for both the LES and highpass filters. The most comprehensive work on the calibration of the model constant for the VMS approach is however the study by Meyers and Sagaut [30]. One of the most important results of this research is that the optimal model coefficient strongly depends on the choice of LES and high-pass filter. Moreover, the authors have provided an analytical framework for the evaluation of the optimal model coefficient in the case of convolution filters. As already mentioned, the DG-projection filter is not a convolution filter and therefore special care is required to extend the conclusions of these works. A different approach has been employed by Cocle et al. [31] who have calibrated the model coefficient of the RVM model a-posteriori by performing LES of decaying homogeneous isotropic turbulence at very high Reynolds number. Due to the different high-pass filter employed, similar but different values of the model constant have been obtained as compared to the study of Meyers and Sagaut [30]. Finally, we mention the work of Bricteux et al. [32] on the development of a wall-adaptive local eddy viscosity RVM model that ensures the correct near-wall scaling of the eddy viscosity.

The studies cited above clearly outline that the performance of the VMS approach is strongly influenced by a number of parameters primarily associated with the LES and high-pass filters. Therefore the systematic and robust application of the DG-VMS approach can be improved by analysing these questions in the context of the DG-projection filter as both the LES filter and 
scale-separation operator. We analyse for this purpose the effect of the DG-LES filter on the ideal energy transfer and the validity of the assumption of absence of SGS dissipation acting on the largest resolved scales. The accuracy of the DG-VMS approach and the associated high-pass filter in replicating the ideal energy transfer will then be considered with the objective of identifying guidelines for the selection of the scale-fraction parameter.

This work is therefore organized as follows. In Section 2 the DG-filter and the ideal DGLES solution are discussed. Their definition is then employed in Sections 3 and 4 to extend the energy transfer analysis framework to the context of DG-LES. The presented framework is then applied in Section 5 to a DNS database of the TGV configuration at $\operatorname{Re}=5000,20000$ and 40000 . Obtained results are then compared in Section 6 to the energy transfer and eddy viscosity provided by the DG-VMS approach. Finally conclusions are presented in Section 7.

\section{The ideal DG-LES solution}

A-priori testing can provide valuable information about the accuracy of LES modeling approaches. The central question with this type of analysis is the definition of an appropriate ideal LES solution, which in the general case is not straightforward. It is, however, essential to answer this question, as the way in which this ideal solution is defined has a direct impact on the way the ideal SGS quantities are computed.

In the classical approach, described in most books on the topic of LES, the LES solution is defined as the spatially filtered DNS solution. For this purpose, convolution filters are conventionally employed such that for any function $f$ we define the filtered function $\bar{f}(x):=$ $\int_{\Omega} G(x-\xi) f(\xi) \mathrm{d} \xi$ with $G(x-\xi)$ being the filter kernel. The convolution filter can be applied to the Navier-Stokes (NS) equations such that the ideal LES solution satisfies the filtered NS equations in their strong form. One of the advantages of this approach is that the application of the convolution filter can be expressed in the Fourier space as $\hat{\bar{f}}(k)=\widehat{G}(k) \hat{f}(k)$ where $\widehat{(\cdot)}$ denotes the Fourier transform. It is thus easy to demonstrate that the convolution filter commutes with the spatial derivatives. This approach however entirely ignores the details of the discretization employed and the fact that the LES solution so defined might not be an admissible solution of the considered discrete problem. It must also be stressed that in most practical LES no explicit filtering is employed and practitioners rely on the implicit filtering introduced by the numerical discretization itself. Under these conditions, most of the assumptions employed by this methodology, including those related to the regularity of the resolved and unresolved scales, are not satisfied. For these reasons, this approach presents serious limitations when extending the results of theoretical studies to FV and FE type methods, which might lead to significant differences between a-priori and a-posteriori analyses.

A second approach has been proposed by Pope [33], in which the LES solution is conceived as the projection of the DNS solution onto a set of local basis functions. It has been shown by Vreman [34] that an arbitrary orthogonal projection operator can be reformulated as a kernel filter such that for any function $f$ we can indicate its projection as $f_{h}(x):=\mathbb{P}_{h}[f]=\int_{\Omega} K_{p}(x, \xi) f(\xi) \mathrm{d} \xi$. The use of a non uniform filter kernel $K_{p}(x, \xi)$ implies that in general the projection operation and differentiation do not commute. For this reason the closure problem needs to be redefined employing the semidiscrete weak form of the equations as described by Pope [33] and in the context of the VMS approach (e.g. [35]).

This methodology provides a definition of the ideal resolved field which is consistent with the employed numerical discretization. Following this approach Beck et al. [36] have defined the 
ideal DG-LES solution as the $L^{2}$-projection of the DNS solution on the discretization space and identified the ideal subgrid stress to develop a Deep Neural Network turbulence model. Similarly van der Bos and Geurts [37] have defined the ideal DG-LES solution by means of a face-based projection to perform a systematic analysis of computational errors of DG-FEM for LES.

The use of a projection type filter however can introduce significant aliasing errors at wavenumbers close to the grid cut-off, producing unphysical reference data. This is a direct consequence of the approximation properties of polynomial basis functions (see [38]).

In this work we propose to employ an alternative approach in which the ideal DG-LES solution is defined as the result of the application of two successive filtering operations. A first convolution filter is applied to the DNS data which filters out wavenumbers beyond the LES grid cut-off. Next, a $L^{2}$-projection of this filtered field is performed on the $h p$-discretization space (referred to in the following as DG-projection). This procedure reduces considerably the aliasing errors introduced by Pope's approach, while allowing the inclusion in the analysis of the effect of the $h p$-discretization associated with the adopted numerical method. A similar approach has been employed by Carati et al. [39] and by Winckelmans et al. [40] to take into account both a smooth filter and the discretization operator in the definition of the LES equations under the assumption that the two operators are commutative.

The following section provides a formal framework for the definition of the ideal DG-LES solution as described above and the expression of the corresponding ideal SGS energy transfer.

\section{The DG-LES framework and the ideal energy transfer}

The N-S equations for an incompressible flow read

$$
\begin{gathered}
\frac{\partial \mathbf{u}}{\partial t}+\boldsymbol{\nabla} \cdot \mathcal{F}_{c}(\mathbf{u}, q)+\boldsymbol{\nabla} \cdot \mathcal{F}_{v}(\mathbf{u}, \boldsymbol{\nabla} \mathbf{u})=0, \quad \forall \mathbf{x} \in \Omega, t \geq 0, \\
\boldsymbol{\nabla} \cdot \mathbf{u}=0,
\end{gathered}
$$

where $\mathbf{u}$ is the velocity field, $q$ is the pressure, and $\mathcal{F}_{c}$ and $\mathcal{F}_{v}$ are the convective and viscous fluxes, defined respectively as

$$
\begin{aligned}
\mathcal{F}_{c}(\mathbf{u}, q) & =\mathbf{u} \otimes \mathbf{u}+q I, \\
\mathcal{F}_{v}(\mathbf{u}, \boldsymbol{\nabla} \mathbf{u}) & =v\left(\boldsymbol{\nabla} \mathbf{u}+(\boldsymbol{\nabla} \mathbf{u})^{T}\right) .
\end{aligned}
$$

We define $\Omega_{h}$ to be a shape regular partition of $\Omega$ into $N$ non-overlapping, non-empty elements of characteristic size $h$ and we further define the broken Sobolev space $S_{h}^{p}:=\left\{\phi \in L^{2}\left(\Omega_{h}\right)\right.$ : $\left.\left.\phi\right|_{K} \in \mathcal{P}^{p}(K), \forall K \in \Omega_{h}\right\}$ to be the space of piecewise polynomials of partial degree at most $p$. Then we indicate as $f_{h}:=\mathbb{P}_{S_{h}^{p}}[f]$ the projection of any function $f$ on the $h p$-discretization defined by the space $S_{h}^{p}$.

Following the approach described in the previous section, we define the ideal DG-LES solution as $\overline{\mathbf{u}}_{h}:=\mathbb{P}_{S_{h}^{p}}[\overline{\mathbf{u}}]$, which is the result of the successive application to the velocity field $\mathbf{u}$ of a convolution filter and the DG-projection filter defined by the space $S_{h}^{p}$. The convolution filter employed in this work is a sharp spectral anisotropic filter with expression in spectral space $\hat{G}(\mathbf{k})=H\left(k_{\mathrm{DG}}-\|\mathbf{k}\|_{\infty}\right)$ where $k_{\mathrm{DG}}=\pi(p+1) / h$ and $H$ is the Heaviside function. ${ }^{1}$

\footnotetext{
${ }^{1}$ The sensitivity of the obtained results to the introduction of the convolution filter is discussed in Appendix C.
} 
Applying the convolution and DG-projection filter to Eq. (1), we derive the evolution equations for the ideal DG-LES solution

$$
\begin{aligned}
\frac{\partial}{\partial t} \int_{\Omega_{h}} \overline{\mathbf{u}}_{h} \phi \mathrm{d} \mathbf{x} & +\sum_{K}\left[\int_{\partial K} \overline{\mathcal{F}}_{c}(\mathbf{u}, q) \cdot \mathbf{n}^{+} \phi^{+} \mathrm{d} \sigma-\int_{K} \overline{\mathcal{F}}_{c}(\mathbf{u}, q) \cdot \boldsymbol{\nabla} \phi \mathrm{d} \mathbf{x}\right. \\
& \left.+\int_{\partial K} \overline{\mathcal{F}}_{v}(\mathbf{u}, \boldsymbol{\nabla} \mathbf{u}) \cdot \mathbf{n}^{+} \phi^{+} \mathrm{d} \sigma-\int_{K} \overline{\mathcal{F}}_{v}(\mathbf{u}, \boldsymbol{\nabla} \mathbf{u}) \cdot \boldsymbol{\nabla} \phi \mathrm{d} \mathbf{x}\right]=0, \quad \forall \phi \in S_{h}^{p},
\end{aligned}
$$

where we have used the commutation property of the convolution filter with spatial derivatives and the definition of the $L^{2}$-projection, which implies, $\int_{\Omega_{h}}\left(\overline{\mathbf{u}}-\overline{\mathbf{u}}_{h}\right) \phi=0, \forall \phi \in S_{h}^{p}$.

The DG-LES equations can now be defined by rewriting Eq. (5) as

$$
\begin{gathered}
\frac{\partial}{\partial t} \int_{\Omega_{h}} \overline{\mathbf{u}}_{h} \phi \mathrm{d} \mathbf{x}+\overbrace{\sum_{K}\left[\int_{\partial K} \mathbf{h}_{c}\left(\overline{\mathbf{u}}_{h}^{+}, \bar{q}_{h}^{+}, \overline{\mathbf{u}}_{h}^{-}, \bar{q}_{h}^{-}, \mathbf{n}^{+}\right) \phi^{+} \mathrm{d} \sigma-\int_{K} \mathcal{F}_{c}\left(\overline{\mathbf{u}}_{h}, \bar{q}_{h}\right) \cdot \boldsymbol{\nabla} \phi \mathrm{d} \mathbf{x}\right]}^{\mathcal{L}_{c}\left(\overline{\mathbf{u}}_{h}, \bar{q}_{h}, \phi\right)}+ \\
\underbrace{\sum_{K}\left[\int_{\partial K} \mathbf{h}_{v}\left(\overline{\mathbf{u}}_{h}^{+}, \overline{\mathbf{u}}_{h}^{-}, \mathbf{n}^{+}\right) \phi^{+} \mathrm{d} \sigma-\int_{K} \mathcal{F}_{v}\left(\overline{\mathbf{u}}_{h}, \boldsymbol{\nabla} \overline{\mathbf{u}}_{h}\right) \cdot \boldsymbol{\nabla} \phi \mathrm{d} \mathbf{x}\right]}_{v \mathcal{L}_{v}\left(\overline{\mathbf{u}}_{h}, \phi\right)}+\mathcal{R}\left(\mathbf{u}, \overline{\mathbf{u}}_{h}, \phi\right)=0, \quad \forall \phi \in S_{h}^{p},
\end{gathered}
$$

where $\mathbf{h}_{c}$ and $\mathbf{h}_{v}$ are the convective and diffusive numerical fluxes and $f^{+}$and $f^{-}$indicate the trace of any function $f$ on the element's boundary $\partial K$.

In Eq. (6), $\mathcal{R}\left(\mathbf{u}, \overline{\mathbf{u}}_{h}, \phi\right)$ is the total DG-LES residual representing the effect of the unresolved scales $\mathbf{u}-\overline{\mathbf{u}}_{h}$ on the resolved field, which can be obtained by comparing Eq. (5) and Eq. (6).

Note that, as the DG-projection filter does not commute with the spatial derivation, in general both the pressure and viscous terms contribute to the total DG-LES residual. In this work, however, we assume that this term is dominated by convective effects thus the contribution of the viscous and pressure terms is neglected ${ }^{2}$. This leads to the following form for the DG-LES residual,

$$
\begin{aligned}
\mathcal{R}\left(\mathbf{u}, \overline{\mathbf{u}}_{h}, \phi\right) \approx \sum_{K} & {\left[\int_{K}\left(\mathcal{F}_{c}\left(\overline{\mathbf{u}}_{h}\right)-\overline{\mathcal{F}}_{c}(\mathbf{u})\right) \cdot \boldsymbol{\nabla} \phi \mathrm{d} \mathbf{x}\right.} \\
& \left.-\int_{\partial K}\left(\mathbf{h}_{c}\left(\overline{\mathbf{u}}_{h}^{+}, \overline{\mathbf{u}}_{h}^{-}, \mathbf{n}^{+}\right)-\overline{\mathcal{F}}_{c}(\mathbf{u}) \cdot \mathbf{n}^{+}\right) \phi^{+} \mathrm{d} \sigma\right] .
\end{aligned}
$$

We point out that, for the derivation of Eq. (7), the sole property which has been required of the spatial filter is commutativity with spatial derivation. If a convolution filter is considered which presents additional regularity properties it is of interest to distinguish, in the total LES

\footnotetext{
${ }^{2}$ The viscous contribution to the DG-LES residual might be relevant for wall-bounded flows. We remark however that, for the incompressible flows considered in this work, the viscous contribution appears due to the non-commutability between the DG-LES filter and spatial derivation and not due to non-linear effects such as those encountered in compressible flows.
} 
residual, the contributions only due to the application of the spatial filter, i.e. subfilter stresses, and those due to the projection on the solution space, i. e. subgrid stresses, see e. g. [39, 40]. A possible approach to decompose the total DG-LES residual in subfilter scale (SFS) and subgrid scale contributions is described in Appendix A.

However, the LES formulation on which DG-VMS simulations are based $[2,41]$ is derived by directly projecting the NS equations onto the DG functional space, which implicitly defines the filter. This operation leads to the appearance of the SGS residual, which represents the full residual term that needs to be model. Additionally, the sharp cut-off filter considered in this work does not present the additional regularity properties which are required for the mathematical analysis of LES seen as a regular convolution.

Therefore, by analogy with the terminology used in the context of DG-VMS simulations and with a slight abuse of notation, in what follows, we will refer to the total DG-LES residual simply as SGS residual.

Indicating as $\left\{\psi_{K}^{1} \ldots \psi_{K}^{N_{p}}\right\} \in \mathcal{P}^{p}(K)$ an orthonormal basis for $\mathcal{P}^{p}(K)$ with $\psi_{K}^{i}(\mathbf{x})=0, \forall \mathbf{x} \in$ $K^{\prime}, K^{\prime} \neq K$, the solution $\overline{\mathbf{u}}_{h}$ is expressed as a linear combination of the basis functions such that

$$
\overline{\mathbf{u}}_{h}(\mathbf{x}, t)=\sum_{K} \sum_{i=1}^{N_{p}} \widetilde{\overline{\mathbf{u}}}_{h}^{i, K}(t) \psi_{K}^{i}(\mathbf{x}), \quad \forall \mathbf{x} \in \Omega_{h} .
$$

The modal coefficients $\widetilde{\overline{\mathbf{u}}}_{h}^{i, K}$ obey the following equation

$$
\frac{\partial \widetilde{\overline{\mathbf{u}}}_{h}^{i, K}}{\partial t}+\mathcal{L}_{c}\left(\overline{\mathbf{u}}_{h}, \bar{q}_{h}, \psi_{K}^{i}\right)+v \mathcal{L}_{v}\left(\overline{\mathbf{u}}_{h}, \psi_{K}^{i}\right)+\mathcal{R}\left(\mathbf{u}, \overline{\mathbf{u}}_{h}, \psi_{K}^{i}\right)=0, \quad \forall K \in \Omega_{h}, \forall i=1 \ldots N_{p},
$$

which is derived from Eq. (6) considering $\psi_{K}^{i}$ as test function and using the orthonormality of the basis (the mass matrix being the identity). These equations can be combined to rewrite the semidiscrete DG-LES equations (6) as

$$
\frac{\partial \overline{\mathbf{u}}_{h}}{\partial t}+\mathrm{L}_{c}\left(\overline{\mathbf{u}}_{h}, \bar{q}_{h}\right)+v \mathrm{~L}_{v}\left(\overline{\mathbf{u}}_{h}\right)+\mathrm{R}\left(\mathbf{u}, \overline{\mathbf{u}}_{h}\right)=0
$$

where

$$
\begin{aligned}
\mathrm{L}_{c} & :=\sum_{K} \sum_{i} \mathcal{L}_{c}\left(\overline{\mathbf{u}}_{h}, \bar{q}_{h}, \psi_{K}^{i}\right) \psi_{K}^{i}, \\
\mathrm{~L}_{v} & :=\sum_{K} \sum_{i} \mathcal{L}_{v}\left(\overline{\mathbf{u}}_{h}, \psi_{K}^{i}\right) \psi_{K}^{i}, \\
\mathrm{R} & :=\sum_{K} \sum_{i} \mathcal{R}\left(\mathbf{u}, \overline{\mathbf{u}}_{h}, \psi_{K}^{i}\right) \psi_{K}^{i} .
\end{aligned}
$$

This leads to the following equation for the evolution of the energy associated to each wavenumber $\mathbf{k}$ of the resolved scales as

$$
\frac{\partial E(\mathbf{k})}{\partial t}+\widehat{\overline{\mathbf{u}}}_{h}(\mathbf{k}) \cdot \widehat{\mathrm{L}}_{c}(\mathbf{k})+v \widehat{\overline{\mathbf{u}}}_{h}(\mathbf{k}) \cdot \widehat{\mathrm{L}}_{v}(\mathbf{k})+\widehat{\overline{\mathbf{u}}}_{h}(\mathbf{k}) \cdot \widehat{\mathrm{R}}(\mathbf{k})=0
$$

The ideal energy transfer from the resolved modes of wavenumber $k$ to all unresolved scales can therefore be obtained from the subgrid residual as

$$
T_{s g s}(k)=\sum_{\|\mathbf{k}\|=k} \widehat{\overline{\mathbf{u}}}_{h}(\mathbf{k}) \cdot \widehat{\mathrm{R}}(\mathbf{k})
$$


Positive values of $T_{s g s}$ correspond to kinetic energy being transferred from resolved to unresolved scales, whereas negative values correspond to energy being transferred from unresolved to resolved scales, commonly indicated as backscatter.

Note that the use of the DG-projection filter introduces discontinuities in the filtered velocity field that need to be taken into account. It also requires the definition of the numerical flux $\mathbf{h}_{c}$ that appears in the surface integral in Eq. (7). The subgrid stress thus depends in general on both, the definition of the filter and the choice of this numerical flux. While this choice might appear arbitrary, it reflects the notion that the subgrid term which needs to be modelled must take into account the choice of the numerical discretization and the numerical dissipation thereby introduced.

In the a-priori analyses presented in the following, we are interested in investigating the ideal SGS dissipation in absence of dissipation introduced by the discretization of the convective flux. For this purpose we consider a central flux $\mathbf{h}_{c}\left(\overline{\mathbf{u}}_{h}^{+}, \overline{\mathbf{u}}_{h}^{-}, \mathbf{n}^{+}\right)=\frac{1}{2}\left(\mathcal{F}_{c}\left(\overline{\mathbf{u}}_{h}^{+}\right)+\mathcal{F}_{c}\left(\overline{\mathbf{u}}_{h}^{-}\right)\right) \cdot \mathbf{n}^{+}$. The stabilizing effect provided by the upwind component of any convective flux can then be separately analysed by means of the same methodology employed in this research for the DG-VMS approach. This approach can be employed to assess the accuracy by which the numerical dissipation can model the SGS stresses, as commonly done in the framework of Implicit LES [42].

\subsection{The modal energy transfer and eddy viscosity}

The analysis presented up to this point represents an extension of the classical energy transfer spectral/Fourier analysis. In the context of the DG method useful information can be extracted by performing this analysis in the modal/polynomial space. For this purpose, let us consider now $\Omega$ to be a cubic domain with $\Omega_{h}$ being a uniform Cartesian grid. We further consider a basis for $S_{h}^{p}$ which is formed by the tensor product of normalized Legendre polynomials of maximum degree $p$. We indicate as $\psi_{K}^{\mathbf{m}}$ the generic element of this basis such that $\mathbf{m}=\left(m_{x}, m_{y}, m_{z}\right)$ and

$$
\psi_{K}^{\mathbf{m}}=l^{m_{x}}\left(\frac{x-x_{K, c}}{h / 2}\right) l^{m_{y}}\left(\frac{y-y_{K, c}}{h / 2}\right) l^{m_{z}}\left(\frac{z-z_{K, c}}{h / 2}\right)
$$

where $x_{K, c}, y_{K, c}$ and $z_{K, c}$ are the coordinates of the barycenter of $K$ and $l^{i}$ is the $i$-th Legendre polynomial normalized such that $\left\|\psi_{K}^{\mathbf{m}}\right\|_{L^{2}\left(\Omega_{h}\right)}=1$.

We then define $W_{h}^{m}=\operatorname{span}\left\{\psi_{K}^{\mathbf{m}}, \forall K \in \Omega_{h}, m-\frac{1}{2}<\|\mathbf{m}\| \leq m+\frac{1}{2}\right\}$, we call $m$ the mode-number and define $\mathbb{P}_{W_{h}^{m}}\left[\overline{\mathbf{u}}_{h}\right]$ as the component of the DG-LES solution $\overline{\mathbf{u}}_{h}$ of mode-number $m$ and the modal energy spectrum as

$$
\tilde{E}(m)=\frac{1}{2} \int_{\Omega_{h}} \mathbb{P}_{W_{h}^{m}}\left[\overline{\mathbf{u}}_{h}\right] \cdot \mathbb{P}_{W_{h}^{m}}\left[\overline{\mathbf{u}}_{h}\right] \mathrm{d} \mathbf{x} .
$$

As $\int_{\Omega_{h}}\left(\mathbb{P}_{W_{h}^{m}}\left[\overline{\mathbf{u}}_{h}\right]-\overline{\mathbf{u}}_{h}\right) \phi \mathrm{d} \mathbf{x}=0, \forall \phi \in W_{h}^{m}$ and $W_{h}^{m} \subset S_{h}^{p}$, from Eq. (5) we can write

$$
\int_{\Omega_{h}} \frac{\partial}{\partial t} \mathbb{P}_{W_{h}^{m}}\left[\overline{\mathbf{u}}_{h}\right] \phi \mathrm{d} \mathbf{x}+\mathcal{L}_{c}\left(\overline{\mathbf{u}}_{h}, \phi\right)+v \mathcal{L}_{v}\left(\overline{\mathbf{u}}_{h}, \phi\right)+\mathcal{R}\left(\mathbf{u}, \overline{\mathbf{u}}_{h}, \phi\right)=0, \quad \forall \phi \in W_{h}^{m},
$$

and it can be immediately obtained that

$$
\frac{\partial \tilde{E}(m)}{\partial t}+\mathcal{L}_{c}\left(\overline{\mathbf{u}}_{h}, \mathbb{P}_{W_{h}^{m}}\left[\overline{\mathbf{u}}_{h}\right]\right)+v \mathcal{L}_{v}\left(\overline{\mathbf{u}}_{h}, \mathbb{P}_{W_{h}^{m}}\left[\overline{\mathbf{u}}_{h}\right]\right)+\mathcal{R}\left(\mathbf{u}, \overline{\mathbf{u}}_{h}, \mathbb{P}_{W_{h}^{m}}\left[\overline{\mathbf{u}}_{h}\right]\right)=0 .
$$


Thus the modal energy transfer from the resolved scales of mode-number $m$ to the unresolved scales can be evaluated as

$$
\widetilde{T}_{s g s}(m):=\mathcal{R}\left(\mathbf{u}, \overline{\mathbf{u}}_{h}, \mathbb{P}_{W_{h}^{m}}\left[\overline{\mathbf{u}}_{h}\right]\right) .
$$

It is can be easily shown that Eq. (20) can be rewritten as

$$
\widetilde{T}_{s g s}(m)=\sum_{K \in \Omega_{h}} \sum_{m-\frac{1}{2}<\|\mathbf{m}\| \leq m+\frac{1}{2}}{\widetilde{\mathbf{u}_{h}}}_{h}^{\mathbf{m}, K} \cdot \mathcal{R}\left(\mathbf{u}, \overline{\mathbf{u}}_{h}, \psi_{K}^{\mathbf{m}}\right),
$$

with $\widetilde{\overline{\mathbf{u}}}_{h}^{\mathbf{m}, K}=\int_{\Omega_{h}} \overline{\mathbf{u}}_{h} \psi_{K}^{\mathbf{m}} \mathrm{d} \mathbf{x}$, which closely resembles Eq. (15) obtained in Fourier space. We thus introduce the concept of a modal eddy viscosity, defined as

$$
\tilde{v}_{e}(m):=\frac{\mathcal{R}\left(\mathbf{u}, \overline{\mathbf{u}}_{h}, \mathbb{P}_{W_{h}^{m}}\left[\overline{\mathbf{u}}_{h}\right]\right)}{\mathcal{L}_{v}\left(\overline{\mathbf{u}}_{h}, \mathbb{P}_{W_{h}^{m}}\left[\overline{\mathbf{u}}_{h}\right]\right)},
$$

which can be interpreted as an additional eddy viscosity which acts on the resolved modes similarly to the spectral eddy viscosity defined by Kraichnan [7].

We point out that neither the modal energy transfer nor the modal eddy viscosity necessarily have a physical meaning. This methodology is however useful as it can be directly compared to the VMS approach and LES models based on a spectral vanishing viscosity such as that proposed by Karamanos and Karniadakis [43]. In the context of DG methods, these approaches are based on modifying the modal energy transfer or eddy viscosity provided by an SGS model as a function of $\mathbf{m}$, see e. g. [44]. A similar energy transfer analysis has already been employed by Oberai et al. [45] to perform a-priori analyses of the VMS approach based on a FE method.

In the following, the modal eddy viscosity is presented normalized as

$$
\tilde{v}_{e}^{\dagger}(m):=\frac{\tilde{v}_{e}(m)}{\sqrt{E\left(\tilde{k}_{\mathrm{DG}}\right) / \tilde{k}_{\mathrm{DG}}}} .
$$

where $E(k)$ is the energy spectrum of the DG-LES field and $\tilde{k}_{\mathrm{DG}}=\frac{(p+1) n_{e l}}{3}$ is a relevant frequency, as will be seen in Sec. 5 .

We point out that Eq. (22) depends on the discretization of the viscous terms. Therefore it provides the modal eddy viscosity which must be provided by the SGS model employing a chosen discretization. This approach highlights the relevance of taking into account the dissipation properties of the numerical scheme used for the discretization of the model term. In Sec. 5 and 6 results will be presented which are based on the BR1 scheme [46] and the BR2 scheme [47].

\section{The DG-LES modelling and the DG-VMS approach}

Starting from Eq. (6), the effect of the subgrid scales can be approximated by a model term that depends only on the resolved field

$$
\mathcal{R}\left(\mathbf{u}, \overline{\mathbf{u}}_{h}, \phi\right) \approx \mathcal{L}_{m}\left(\overline{\mathbf{u}}_{h}, \phi\right)
$$

One common approach to formulate SGS models for DG methods is to discretize LES models derived in the continuous framework, such as those relying on an eddy-viscosity approach like the Smagorinsky model. 
For the Smagorinsky model, a SGS flux is introduced in the filtered NS equations which takes the form

$$
\mathcal{F}_{m}=2 v_{s}(\boldsymbol{\nabla u}) S(\boldsymbol{\nabla} \mathbf{u}) \quad \text { with } \quad v_{s}(\boldsymbol{\nabla} \mathbf{u})=\left(C_{s} \Delta\right)^{2}\|S(\boldsymbol{\nabla u})\|,
$$

where $S=\frac{1}{2}\left(\nabla \mathbf{u}+\nabla \mathbf{u}^{T}\right)$ is the strain rate tensor, with norm $\|S\|=\sqrt{2 S_{i j} S_{i j}}$. The Smagorinsky constant $C_{s}$ has values between 0.1 and 0.2 , and $\Delta$ is the filter width which in the DG framework can be defined as $\Delta=h /(p+1)$.

By applying the same numerical treatment used for the viscous fluxes, the DG-LES model term can be written as,

$$
\mathcal{L}_{m}\left(\overline{\mathbf{u}}_{h}, \phi\right)=\sum_{K}\left[\int_{\partial K} \mathbf{h}_{m}\left(\overline{\mathbf{u}}_{h}^{+}, \overline{\mathbf{u}}_{h}^{-}, \mathbf{n}^{+}\right) \phi^{+} \mathrm{d} \sigma-\int_{K} \mathcal{F}_{m}\left(\overline{\mathbf{u}}_{h}\right) \boldsymbol{\nabla} \phi \mathrm{d} \mathbf{x}\right], \quad \forall \phi \in S_{h}^{p},
$$

where $\mathbf{h}_{m}$ is the model numerical flux.

Following the approach presented above (see Sec. 3.1), the modelled spectral energy transfer can therefore be evaluated as

$$
T_{m}(k)=\sum_{\|\mathbf{k}\|=k} \widehat{\overline{\mathbf{u}}_{h}}(\mathbf{k}) \cdot \widehat{\mathrm{L}_{m}}(\mathbf{k}) \quad \text { with } \quad \mathrm{L}_{m}:=\sum_{K} \sum_{\mathbf{m}} \mathcal{L}_{m}\left(\overline{\mathbf{u}}_{h}, \psi_{K}^{\mathbf{m}}\right) \psi_{K}^{\mathbf{m}},
$$

and the modelled modal energy transfer and eddy viscosity take the form

$$
\widetilde{T}_{m}(m):=\mathcal{L}_{m}\left(\overline{\mathbf{u}}_{h}, \mathbb{P}_{W_{h}^{m}}\left[\overline{\mathbf{u}}_{h}\right]\right), \quad \text { and } \quad \widetilde{v}_{m}^{\dagger}(m):=\frac{\mathcal{L}_{m}\left(\overline{\mathbf{u}}_{h}, \mathbb{P}_{W_{h}^{m}}\left[\overline{\mathbf{u}}_{h}\right]\right)}{v \mathcal{L}_{v}\left(\overline{\mathbf{u}}_{h}, \mathbb{P}_{W_{h}^{m}}\left[\overline{\mathbf{u}}_{h}\right]\right)} .
$$

The VMS approach is based on the separation of the resolved scales into large and small resolved scales by means of a projection filter. To this end, we separate the solution space into a large-scale space $V^{L}:=\bigcup_{m \leq p_{L}} W_{h}^{m}$ and a small-scale space $V^{S}:=S_{h}^{p} \backslash V^{L}$, where $p_{L}$ is the so-called scale-separation parameter and we indicate as $\beta=\left(p_{L}+1\right) /(p+1)$ the scale-fraction parameter. $^{3}$

The original formulation of the VMS approach proposed by Hughes et al. [15] relies on two assumptions: the absence of energy transfer between the large resolved and the unresolved scales and the fact that the SGS model should be evaluated from the small-resolved scales.

This leads to a model term which takes the form

$$
\left(\boldsymbol{\nabla} \cdot \mathcal{F}_{m}\left(\overline{\mathbf{u}}_{h}\right)\right)_{s-s}=\mathbb{P}_{V^{s}}\left[\boldsymbol{\nabla} \cdot\left(2 v_{s}\left(\mathbb{P}_{V^{s}}\left[\boldsymbol{\nabla} \overline{\mathbf{u}}_{h}\right]\right) S\left(\mathbb{P}_{V^{s}}\left[\boldsymbol{\nabla} \overline{\mathbf{u}}_{h}\right]\right)\right)\right]
$$

This approach is commonly referred to as the small-small approach, as both the eddy viscosity and the strain rate tensor in the model term are computed directly from the small resolved scales. The outer filter operation restricts the action of the LES model only to the small-scale solution corresponding to mode-numbers higher than the scale-separation parameter. It corresponds therefore to the assumption $\widetilde{T}_{s g s}(m) \approx 0$ and $\widetilde{v}_{s g s} \approx 0$ for $m \leq p_{L}$.

We point out once more that the $\mathrm{L}^{2}$-projection and differentiation do not commute, thus the order of the operations is important in the definition of the model term. In particular, we remark that while the effect of the model is applied to the small-scale solution, the model flux is

${ }^{3}$ Other choices can be employed for the definition of the large-scale space. Further discussion on this topic is presented in Appendix D. 
computed from the filtered gradient ${ }^{4}$ which does not correspond to the gradient of the small-scale solution. This distinction disappears in the original formulation employing a convolution filter.

Other variants of the VMS model have been proposed in the literature. They include the large-small [15] and the all-small [25] approaches which correspond to evaluating the eddy viscosity from either the low-pass filtered gradients or all the resolved scales. In the case of homogeneous isotropic turbulence this modification leads to only minor differences and it amounts to modifying the model coefficient [31].

Vreman [1] has proposed to discard the outer filter in Eq. (29) leading to

$$
\left(\boldsymbol{\nabla} \cdot \mathcal{F}_{m}\left(\overline{\mathbf{u}}_{h}\right)\right)_{\mathrm{Vrem}}=\boldsymbol{\nabla} \cdot\left(2 v_{s}\left(\mathbb{P}_{V^{s}}\left[\boldsymbol{\nabla} \overline{\mathbf{u}}_{h}\right]\right) S\left(\mathbb{P}_{V^{s}}\left[\boldsymbol{\nabla} \overline{\mathbf{u}}_{h}\right]\right)\right)
$$

This approach has led to qualitatively similar results to the small-small approach [1]. However it is not consistent with the original formulation by Hughes et al. [15] and is more closely related to the high-pass filtered Smagorinsky model [49]. This formulation has nonetheless the advantage of reducing the number of filtering operations required for the evaluation of the model. A large reduction of its computational cost can therefore be obtained for some formulations of the DG method (e.g. nodal DG).

Chapelier et al. [2] have proposed an all-all approach consisting in evaluating both the eddy viscosity and the strain rate from all resolved scales and retaining only the outer filtering operation

$$
\left(\boldsymbol{\nabla} \cdot \mathcal{F}_{m}\left(\overline{\mathbf{u}}_{h}\right)\right)_{\mathrm{a}-\mathrm{a}}=\mathbb{P}_{V^{s}}\left[\boldsymbol{\nabla} \cdot\left(2 v_{S}\left(\boldsymbol{\nabla} \overline{\mathbf{u}}_{h}\right) S\left(\boldsymbol{\nabla} \overline{\mathbf{u}}_{h}\right)\right)\right] .
$$

This approach is specifically tailored for the DG-modal formulation employing orthonormal hierarchical bases. In this case the outer filtering operation can be implicitly applied by removing the model term from the equation of the modal coefficients associated with the large-scale space basis functions. Thus the all-all approach presents the same computational cost as the standard Smagorinsky model for this class of methods.

In Sec. 6 the three variants of the DG-VMS approach here described are compared by analysing their accuracy in replicating the ideal energy transfer mechanism.

\section{Ideal energy transfer from DNS data}

The methodology laid out in the Sec. 3 is applied to three DNS data sets of the TGV configuration at $\operatorname{Re}=5000,20000$ and 40000 . The reference DNS have been performed using the sixth-order incompressible flow solver Incompact3D [23]. The considered computations have been obtained on a regular Cartesian mesh of respectively $1280^{3}, 3456^{3}$ and $5400^{3}$ nodes in a triperiodic domain of $[-\pi, \pi]^{3}$ using symmetries to divide by 8 the number of degrees of freedom (dofs) actually computed. A snapshot of each of these data sets at $t=14$ (non-dimensional time units) is selected for analysis. At this time the flow is fully developed in a state close to isotropic and homogeneous conditions with values of the Reynolds number based on the Taylor microscale $\operatorname{Re}_{\lambda}=136,286$ and 400 for $\operatorname{Re}=5000,20000$ and 40000 respectively.

In Fig. 1 we report the energy spectrum of the snapshot corresponding to $\mathrm{Re}=5000$. On the same figure we report the energy spectra of the ideal DG-LES solution for $p=7$ and respectively $72^{3}, 144^{3}$ and $288^{3}$ dofs, computed as described in Appendix B.

\footnotetext{
${ }^{4}$ When employing the BR1 and BR2 schemes this requires the use of the filtered lifted derivatives (see e.g. [48]).
} 

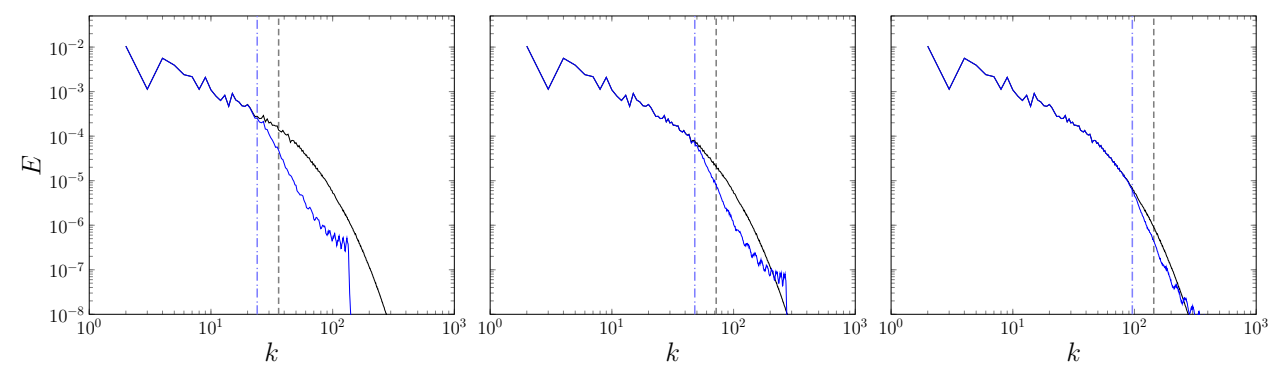

Figure 1: TGV at Re = 5000: Energy spectra from the DNS computation (black) and the ideal DG-LES solution (blue) for various discretizations: $p=7$ and $72^{3}, 144^{3}$ and $288^{3}$ dofs. The corresponding values of $k_{\mathrm{DG}}$ and $\tilde{k}_{\mathrm{DG}}$ are marked by black dashed lines and blue dot-dashed lines respectively.
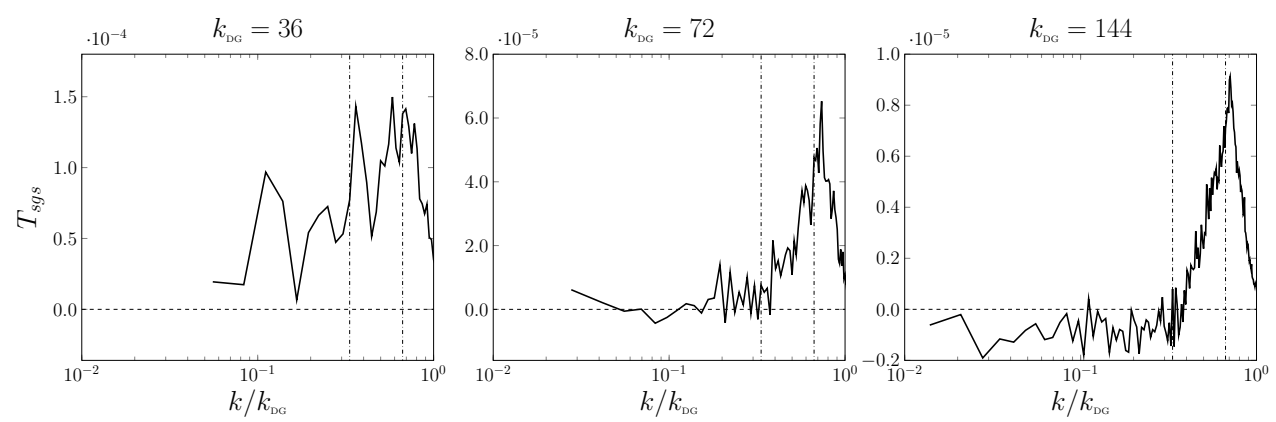

Figure 2: TGV at Re $=5000$ : Ideal SGS dissipation spectrum for three discretizations with $p=7$. The values $\tilde{k}_{\mathrm{DG}}$ and $k_{\mathrm{DG}} / 3$ are marked by dash-dotted lines.

When analysing DG-LES simulation results it is assumed that the resolution limit is defined by the cut-off wavenumber $k_{\mathrm{DG}}=\frac{\pi(p+1)}{h}=\frac{(p+1) n_{e l}}{2}$ (marked by black dashed lines in Fig. 1) where $n_{e l}$ is the number of elements in one direction. By analysing Fig. 1, however, it can be observed that the DG-LES spectrum is almost undistinguishable from that corresponding to the DNS up to a wavenumber $\tilde{k}_{\mathrm{DG}}=\frac{(p+1) n_{e l}}{3}$ and decays rapidly for higher wavenumbers. Moreover, the energy spectrum is "polluted" by the presence of discontinuities for wavenumbers close to $k_{\mathrm{DG}}$. Additionally the discontinuities generate a tail on the energy spectrum that decays as $k^{-2}$. It is argued therefore that $\tilde{k}_{\mathrm{DG}}$ is more relevant in identifying the resolving capabilities of the DG discretization. We will see in Sec. 5.2 that these observations are valid for other values of the polynomial degree $p$.

The values of $\tilde{k}_{\mathrm{DG}}$ for the three discretizations considered are therefore also reported in Fig. 1. These wavenumbers fall respectively within the inertial range $\left(E \propto k^{-5 / 3}\right)$, at the end of the inertial range and in the dissipation range.

In Fig. 2 we report the ideal SGS dissipation spectra, as defined in Eq. (15), computed for the three considered resolutions. In each plot, we observe that the dissipation spectrum presents a peak at $\tilde{k}_{\mathrm{DG}}$ and rapidly decays for higher wavenumbers. This behaviour is remarkably different as compared to the case of sharp spectral filters for which a cusp appears at the cut-off wavenumber. This observation further confirms the relevance of $\tilde{k}_{\mathrm{DG}}$ in identifying the resolving capabilities of the employed discretization. 

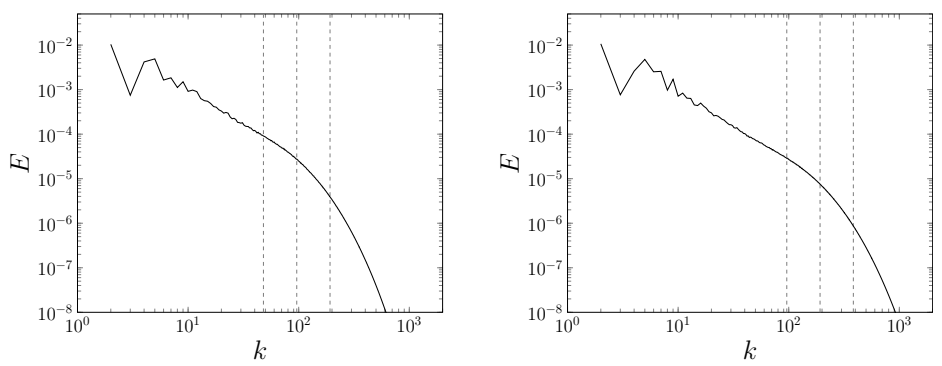

Figure 3: Energy spectra for the TGV at Re $=20000$ (left), 40000 (right). The considered values of $\tilde{k}_{\mathrm{DG}}$ are marked by dashed lines.

As regards the lower wavenumbers, we observe that for the coarsest employed discretization the interaction between the largest-resolved scales and the unresolved scales is non-negligible. As the value of $\tilde{k}_{\mathrm{DG}}$ is moved toward the dissipation range, we observe from the middle and right panel of Fig. 2 a clearly different behaviour. In this case the ideal energy transfer is negligible up to a wavenumber corresponding to $\tilde{k}_{\mathrm{DG}} / 2=k_{\mathrm{DG}} / 3$ and the dissipation spectrum rapidly increases up to $\tilde{k}_{\mathrm{DG}}$ corresponding to a hyperviscous-type behaviour. A sharper peak of the SGS dissipation spectrum is observed as the resolution is increased. Additionally for the finest resolution considered $k_{\mathrm{DG}}=144$ the ideal energy transfer is negative for wavenumbers below $k_{\mathrm{DG}} / 3$ corresponding to backscatter. While similar results are seldom presented in the literature we mention that both Domaradzki [8] and Métais and Lesieur [10] reported negative values of the eddy viscosity in the smallest wavenumber range employing an isotropic sharp spectral filter with cut-off wavenumber in the dissipation range. Moreover Métais and Lesieur [10] have shown that with this type of LES filter a negative value of the plateau of the eddy viscosity is obtained assuming an energy spectrum $E(k) \propto k^{-m}$ with $m \geq 5$.

In order to analyse the generality of these observations we consider now the TGV configuration at higher Reynolds numbers, namely $\mathrm{Re}=20000$ and 40000 . The corresponding energy spectra are reported in Fig. 3 as well as the values of $\tilde{k}_{\mathrm{DG}}$ corresponding to three discretizations considered. These discretizations correspond to $p=7$ and a number of dofs equal to $144^{3}, 288^{3}$ and $576^{3}$ for the lower Reynolds number and $288^{3}, 576^{3}$ and $1152^{3}$ for the higher Reynolds number configuration. For both configurations the coarsest discretizations correspond to $\tilde{k}_{\mathrm{DG}}$ in the inertial range, whereas the finer discretizations correspond respectively to $\tilde{k}_{\mathrm{DG}}$ at the end of the inertial range and $\tilde{k}_{\mathrm{DG}}$ in the dissipation range.

For both configurations and all resolutions considered we observe in Figs. 4 and 5 again a peak of the dissipation spectrum at $\tilde{k}_{\mathrm{DG}}$ and a rapid decay towards $k_{\mathrm{DG}}$, confirming the results obtained for the configuration at $\mathrm{Re}=5$ 000. In this case, however, for the two lower resolutions, with $\tilde{k}_{\mathrm{DG}}$ located in the inertial range, we observe a mixed viscous-hyperviscous behaviour. The viscous type behaviour, corresponding to an ideal SGS dissipation spectrum which scales as $k^{\frac{1}{3}}, 5$ is dominant for the low and intermediate wavenumbers up to approximately $k_{\mathrm{DG}} / 3$ whereas the hyperviscous behaviour is dominant for higher wavenumbers up to $\tilde{k}_{\mathrm{DG}}$.

\footnotetext{
${ }^{5}$ We refer to viscous-type behaviour when the SGS dissipation acts as a viscous dissipation with constant viscosity. In the spectral space, for homogeneous isotropic turbulence, this quantity is proportional to $k^{2} E(k)$, see e.g. [8], and therefore proportional to $k^{2} k^{-5 / 3}=k^{1 / 3}$ in the inertial range.
} 

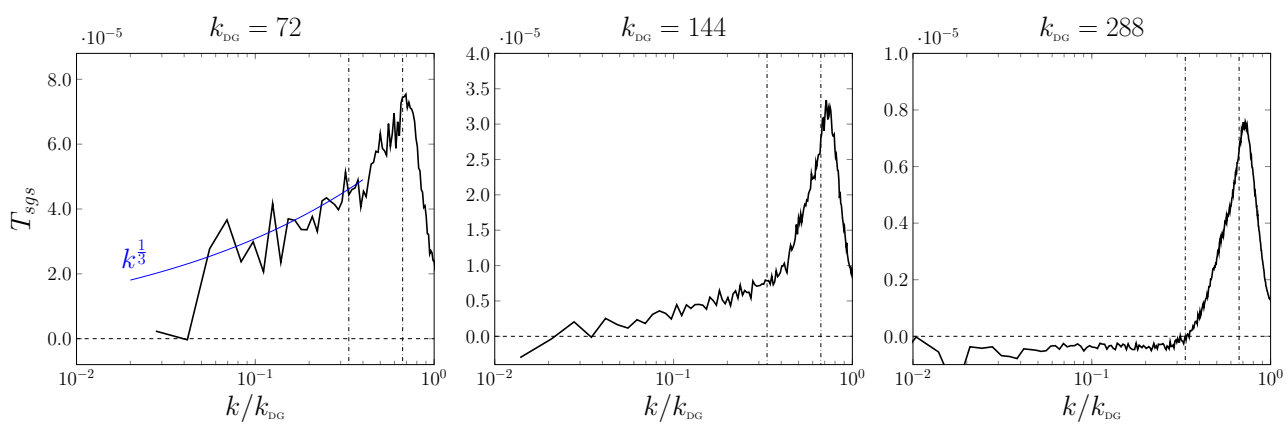

Figure 4: TGV at Re $=20000$ : Ideal SGS dissipation spectrum for three discretizations with $p=7$. The values $\tilde{k}_{\mathrm{DG}}$ and $k_{\mathrm{DG}} / 3$ are marked by dash-dotted lines.
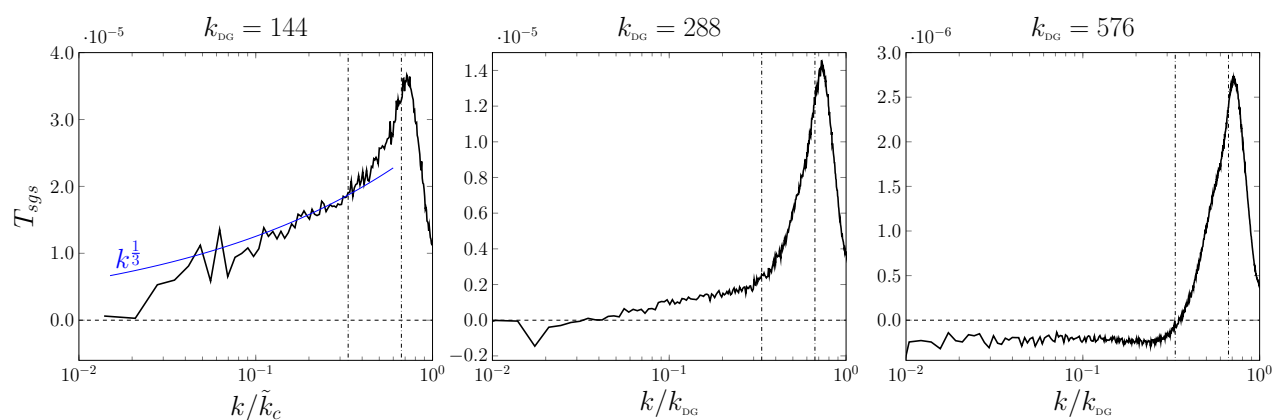

Figure 5: TGV at $\operatorname{Re}=40000$ : Ideal SGS dissipation spectrum for three discretizations with $p=7$. The values $\tilde{k}_{\mathrm{DG}}$ and $k_{\mathrm{DG}} / 3$ are marked by dash-dotted lines.

However, as resolution is increased, the contribution of wavenumbers below $k_{\mathrm{DG}} / 3$ to the total SGS dissipation is progressively reduced and most of the SGS dissipation acts on the wavenumbers $\left[k_{\mathrm{DG}} / 3, k_{\mathrm{DG}}\right]$. Eventually, as the resolution is further increased and $\tilde{k}_{\mathrm{DG}}$ moves into the dissipation range, the interaction between the large-resolved scales and unresolved scales becomes negligible. This can be observed in the right panels of Figs. 4 and 5. In this case, the energy transfer is dominated by the SGS dissipation acting on wavenumbers $\left[k_{\mathrm{DG}} / 3, k_{\mathrm{DG}}\right]$. For wavenumbers below $k_{\mathrm{DG}} / 3$ the energy transfer is predominantly negative corresponding to backscatter.

The results obtained therefore indicate that the large-resolved scales are free of interaction with the unresolved ones only when the DG-LES limit of resolution falls at the end of the inertial range and within the dissipation range. For most cases of practical interest, when a coarser resolution is employed, a mixed viscous-hyperviscous type behaviour can be observed and the SGS dissipation acting on the large-resolved scales is not negligible.

The mixed type behaviour is not observed in Fig. 2 as the TGV at $\operatorname{Re}=5000$ presents a very short inertial range.

\subsection{Ideal modal energy transfer and eddy viscosity}

We now analyse the modal energy transfer as defined by Eq. (20) for the same configurations described in the previous section. Obtained results are reported in Fig. 6. We observe a remarkably consistent behaviour across all resolutions and Reynolds numbers considered. A first region 

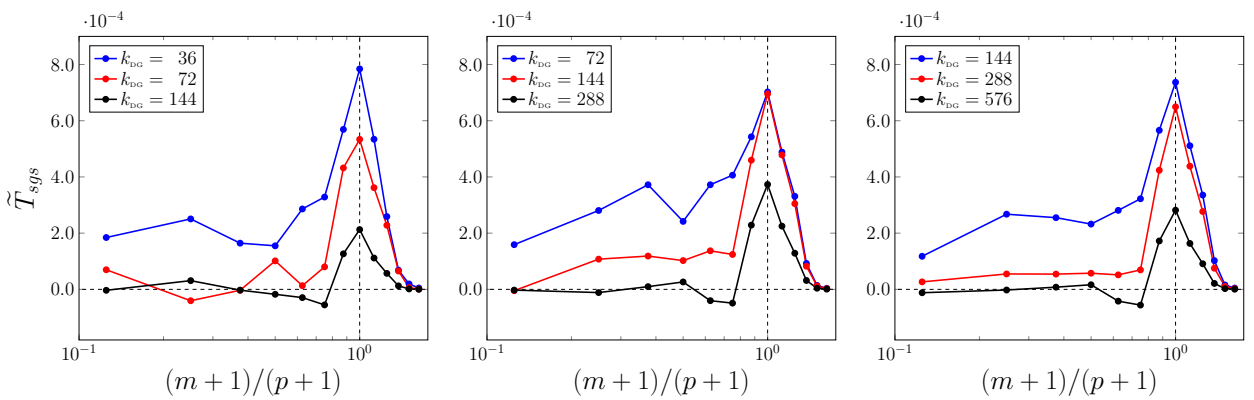

Figure 6: Modal energy transfer for the ideal SGS stress for the TGV at Re $=5000$ (left), 20000 (center), and 40000 (right) for various discretizations with $p=7$.
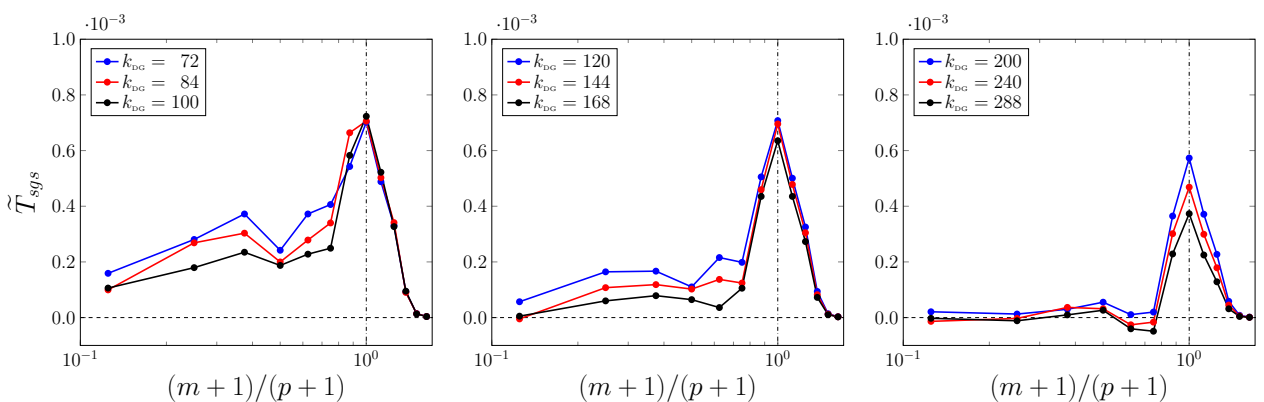

Figure 7: TGV at Re = 20000: Modal energy transfer for the ideal SGS stress for several discretizations with $p=7$.

can be identified which is characterized by a nearly constant modal energy transfer for modenumbers $m$ from 0 to 5. The modal energy transfer then increases rapidly presenting a peak at $m=p$ and then decreases smoothly for higher mode-numbers. We remark that these two different behaviours are separated by the same mode-number corresponding to $(m+1) /(p+1)=0.75$ for all the discretizations and Reynolds numbers considered.

As we would expect from the previous analysis in Fourier space, the energy transferred to modes corresponding to low mode-numbers is not in general negligible. As the discretization is refined this value progressively decreases and the energy transfer mechanism is dominated by the SGS dissipation acting on modes $(m+1) /(p+1)>0.75$.

The consistency of the described behaviour is further illustrated by Fig. 7 which reports the modal energy transfer obtained at $\operatorname{Re}=20000$ for 9 discretizations with $p=7$ and a number of dofs between $144^{3}$ and $576^{3}$. We additionally observe that for relatively coarse discretizations as the resolution is increased, the main effect is to reduce the modal energy transfer at low modenumbers. Only when the resolution limit is in the dissipation range $\left(k_{\mathrm{DG}}>168\right)$ a significant reduction of the peak value is obtained as the discretization is further refined.

The ideal modal eddy viscosity, as defined in Eq. (22) using the BR1 scheme for the viscous discretization, is reported in Fig. 8 for the three Reynolds numbers and discretizations.

Similarly to what has been observed for the modal energy transfer, the modal eddy viscosity presents a plateau at mode-numbers $m \leq 5$ and increases for higher mode-numbers. In contrast to the modal dissipation spectrum, however, the modal eddy viscosity presents in general 

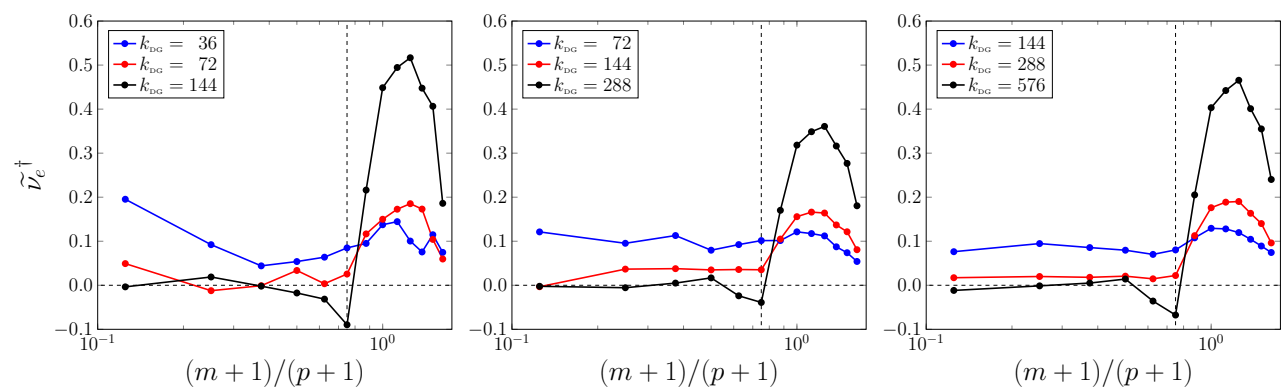

Figure 8: Ideal modal eddy viscosity for the ideal subgrid stress for the TGV at Re $=5000$ (left), 20000 (center), 40000 (right) for various discretizations with $p=7$.

a "parabolic" shape (in place of a spike) with a smoother increase and decay for relatively high mode-numbers.

To conclude this analysis we observe that all the obtained results indicate that the interaction between large-resolved scales and unresolved ones, when employing the DG-LES filter, is negligible only for finite Reynolds numbers when the turbulent scales are resolved up to the beginning of the dissipation range. Based on these findings, it could be argued that models based on this assumption present a limited applicability as they would rely on high resolution being available and thus provide a limited computational gain as compared to (underresolved) DNS. In contrast, for most cases of practical interest, at relatively high Reynolds numbers when the resolution limit falls within the inertial range, the SGS dissipation acting on the large-resolved scales is not negligible. We want to remark however that the resolution requirements in an actual simulation vary in space and time and thus this assumption might be locally valid. This is the case for transitional or spatially inhomogeneous flows.

As an example, we illustrate in Fig. 9 the modal energy transfer for the TGV at $\operatorname{Re}=20000$ at various times for a discretization corresponding the intermediate resolution considered $(p=7$, $n_{e l}=36$ and $288^{3}$ dofs). It can be observed that the SGS dissipation rapidly increases during the transition phase (left panel of Fig. 9) and the energy transfer from large scales remains non negligible during the first part of the decay phase (central panel). However for $t>14$ the resolution is sufficient such that the energy transfer from modes $m \leq 5$ is clearly negligible (right panel). Thus the ideal LES model should be able to adapt to each of these conditions by reducing the SGS model dissipation applied to large scales during the initial transition phase and late dissipation phase.

\subsection{Sensitivity to the polynomial degree}

In this section we investigate the generality of the obtained results by analysing discretizations corresponding to various values of the polynomial degree $p$. All the results here reported have been obtained from a snapshot at $t=14$ of the TGV at $\mathrm{Re}=20000$. The comparisons are carried out by fixing the total number of dofs to the same values employed in Sec. 5, that is $144^{3}$, $288^{3}$ and $576^{3}$ dofs. Four values of the polynomial degree are at first considered: $p=5,7,8$, and 11 .

Fig. 10 presents the energy spectra of the DNS data set and the ideal DG-LES solutions for all considered discretizations. We observe that for a fixed number of dofs the energy spectra are almost identical up to $k_{\mathrm{DG}}$ and as mentioned in the previous section (see Fig. 1) identical to the 

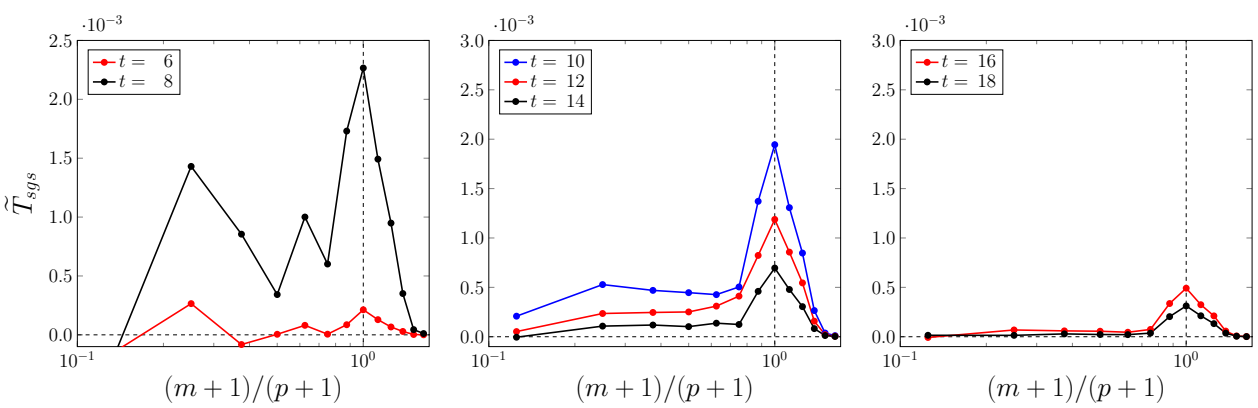

Figure 9: TGV at Re $=20000$ : Ideal modal energy transfer for the ideal SGS stress at various times for $p=7$ and $288^{3}$ dofs.
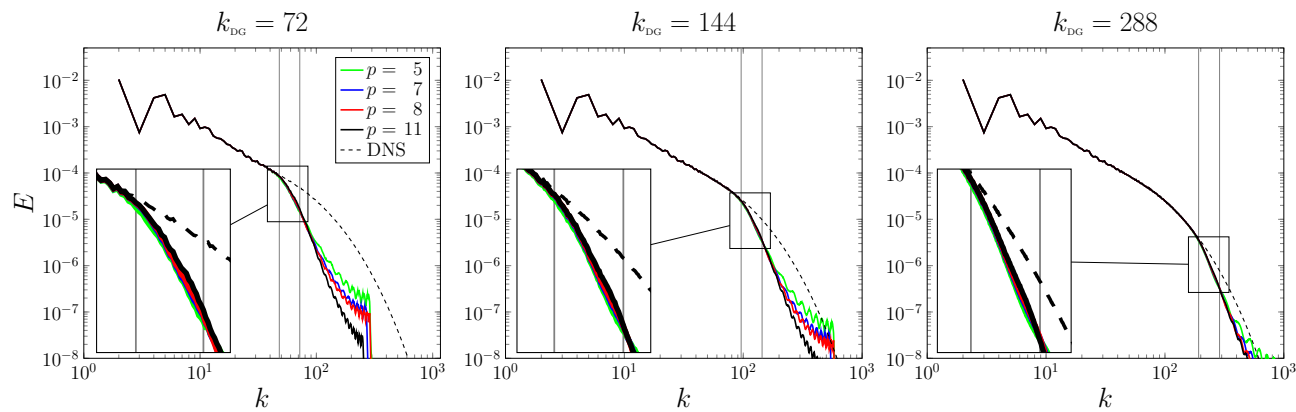

Figure 10: TGV at Re $=20000$ : Energy spectra of the DNS data and the ideal DG-LES solution for various discretizations for $144^{3}, 288^{3}$ and $576^{3}$ dofs. Close-up view at wavenumbers between $\tilde{k}_{\mathrm{DG}}$ and $k_{\mathrm{DG}}$.

DNS spectrum up to $\tilde{k}_{\mathrm{DG}}$. The most notable differences appear in the tail of the spectra related to the discontinuities of the DG-LES solutions. Thus we can reasonably conclude that increasing the polynomial degree for a fixed number of dofs has a limited effect on the resolving capabilities of the DG-LES method. ${ }^{6}$

This conclusion is also confirmed by analysing Fig. 11 which reports the SGS dissipation spectrum. Indeed the same behaviour can be observed for all polynomial degrees confirming the generality of the conclusions drawn in the previous section.

In Figs. 12 and 13 we report the modal energy transfer and eddy viscosity. The modal energy transfer levels cannot be directly compared, as a different number of modes is retained for each polynomial degree, however we can observe that the same trend seen for $p=7$ (see Sec. 5.1) is obtained for the other discretizations. The generality of our conclusions is further illustrated by Fig. 13 which demonstrates the close agreement of the modal eddy viscosity for all discretizations and confirms the relevance of the mode-number $m+1=0.75(p+1)$ in separating the two different behaviours.

We consider now relatively lower polynomial degree representations: $p=2,3,4$ and 5. The energy spectra for all discretizations are not reported here as they lead to the same conclusions

${ }^{6}$ This observation only concerns the accuracy of the considered solution space in representing the DNS solution. It does not take into account the dissipation properties of the numerical fluxes as done $e$. g. by Moura et al. [50]. 

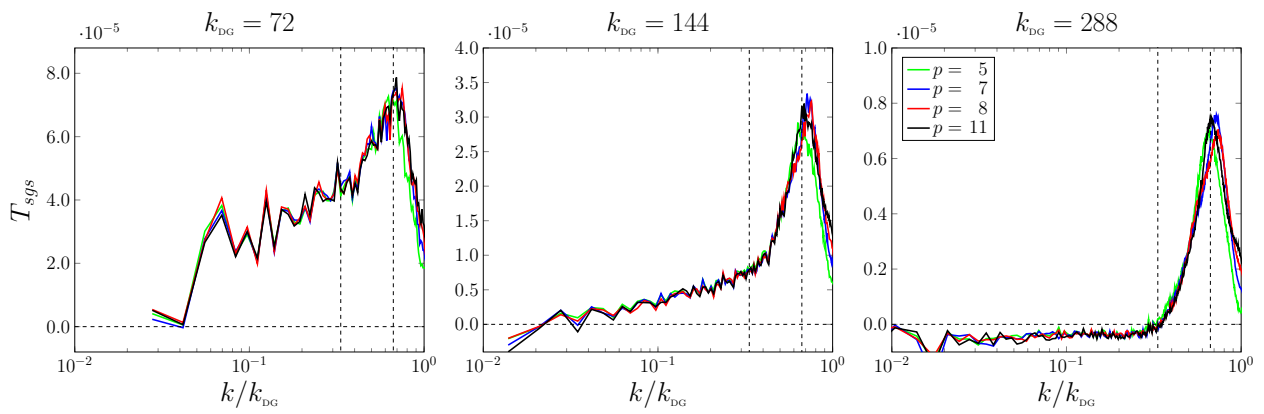

Figure 11: TGV at $\operatorname{Re}=20000$ : Ideal SGS dissipation spectrum for various discretizations for $144^{3}, 288^{3}$ and $576^{3}$ dofs. Dashed lines mark values of $\tilde{k}_{\mathrm{DG}}$ and $k_{\mathrm{DG}} / 3$.
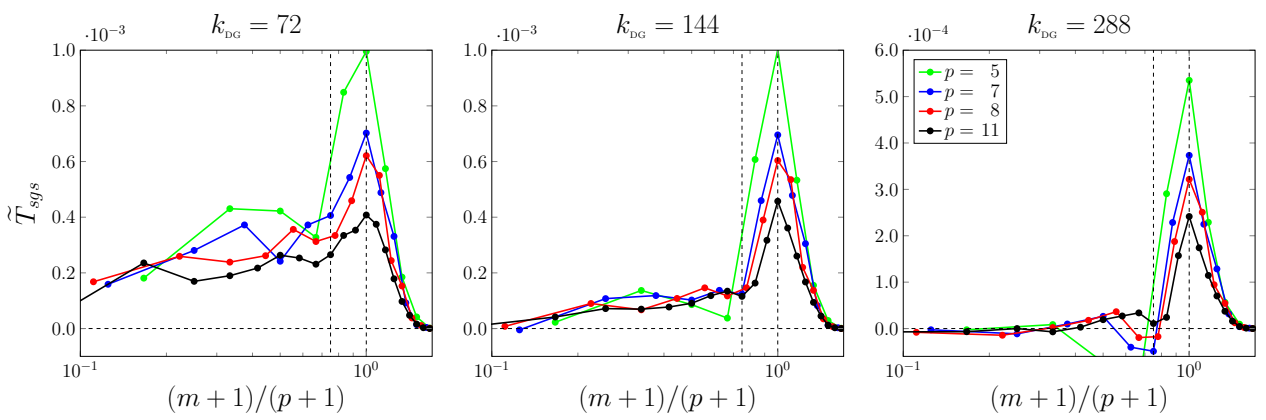

Figure 12: TGV at $\operatorname{Re}=20000$ : Ideal modal energy transfer for various discretizations for $144^{3}, 288^{3}$ and $576^{3}$ dofs. Dashed lines indicate mode-numbers $m+1=0.75(p+1)$ and $m=p$.
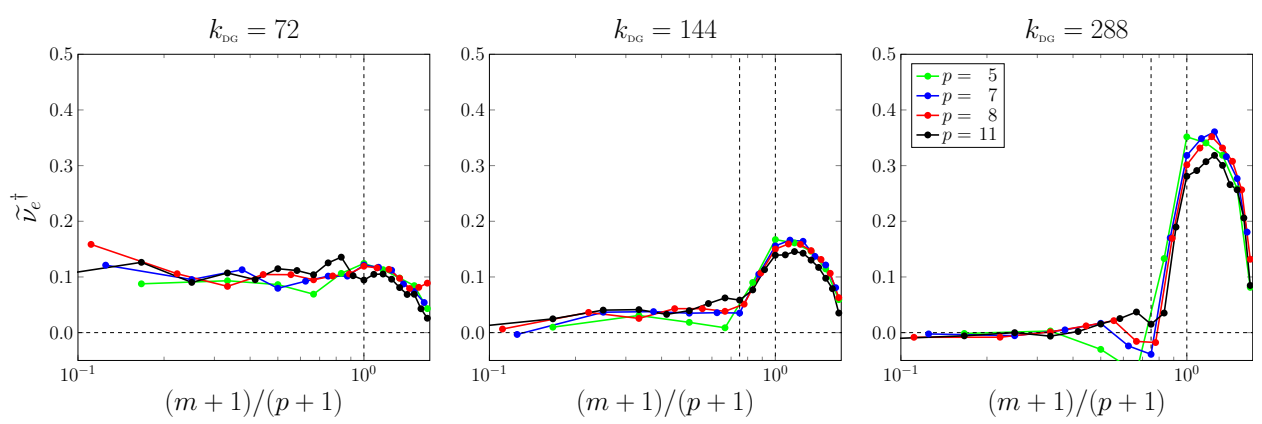

Figure 13: TGV at $\operatorname{Re}=20000$ : Ideal modal eddy viscosity for various discretizations for $144^{3}, 288^{3}$ and $576^{3}$ dofs. Dashed lines indicate mode-numbers $m+1=0.75(p+1)$ and $m=p$. 

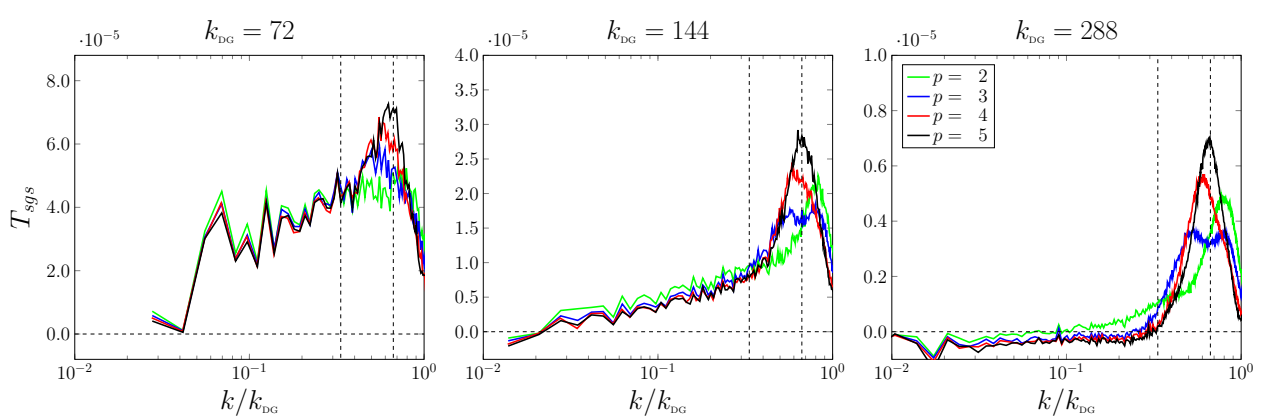

Figure 14: TGV at $\mathrm{Re}=20000$ : Ideal SGS dissipation spectrum for various discretizations for $144^{3}, 288^{3}$ and $576^{3}$ dofs Dashed lines mark values of $\tilde{k}_{\mathrm{DG}}$ and $k_{\mathrm{DG}} / 3$.
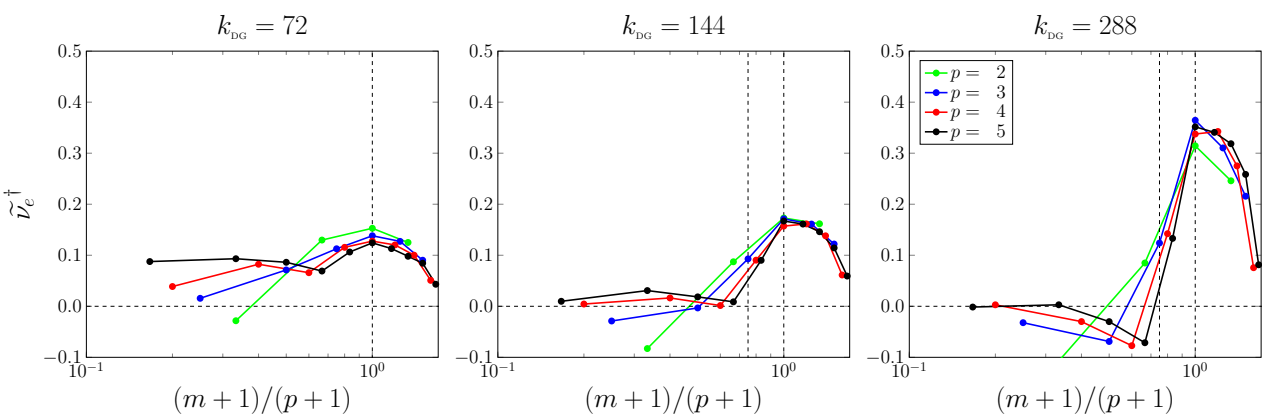

Figure 15: TGV at $\operatorname{Re}=20000$ : Ideal modal eddy viscosity for various discretizations for $144^{3}, 288^{3}$ and $576^{3}$ dofs. Dashed lines indicate mode-numbers $m+1=0.75(p+1)$ and $m=p$.

drawn from Fig. 10. More marked differences can be observed in Figs. 14 and 15 reporting the ideal SGS dissipation spectrum and modal eddy viscosity. Overall a similar sensitivity to the available resolution can be recognized for different values of $p$ in Fig. 14. However, in constrast with the results obtained for higher polynomial degrees, the presence, location and value of the peak of the SGS dissipation spectrum appear to be dependent on the polynomial degree for $p \leq 4$.

Similarly, in Fig. 15 we observe relatively marked differences in the modal eddy viscosity for different values of $p$. As the resolution is increased the SGS dissipation acts on the highest modenumbers. However compared to Fig. 13, no real plateau can be identified for the modal eddy viscosity at low modenumbers and for the highest resolution (right panel of Fig. 15) markedly negative values are obtained for the modal viscosity at low modenumbers.

\section{A-priori analysis of the DG-VMS approach}

In this section, we perform an a-priori analysis of the all-all and Vreman variants of the DGVMS model. For this purpose, we evaluate the DG-VMS model from the ideal DG-LES solution corresponding to $\operatorname{Re}=20000$ and $t=14$ for $p=7$ and $n_{e l}=72$. As described in the previous section, for this Reynolds number and discretization considered the resolution limit, $\tilde{k}_{\mathrm{DG}}=192$, falls within the dissipation range. Under these conditions the interaction between large-resolved 

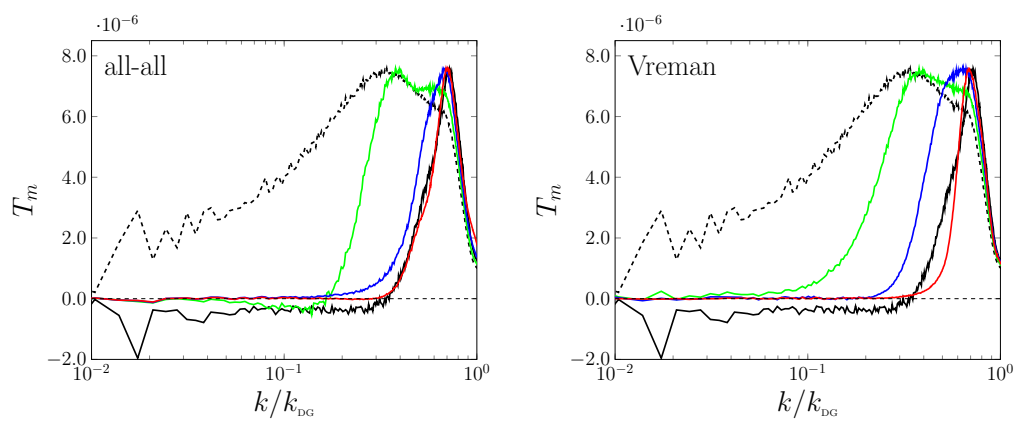

Figure 16: $\mathrm{TGV}$ at $\mathrm{Re}=20000, p=7, k_{\mathrm{DG}}=288$ : Ideal SGS energy transfer (black solid), SGS model dissipation spectrum provided by the Smagorinsky model (dashed) and two variants of the DG-VMS approach for: $\beta=0.25$ (green), $\beta=0.5$ (blue), and $\beta=0.75$ (red) using the BR2 scheme $\left(\eta_{b r 2}=2\right)$.

and unresolved scales is negligible (see Fig. 4) and we expect the DG-VMS approach to recover the ideal SGS dissipation.

Both variants of the DG-VMS model are evaluated from this solution for different values of the scale fraction parameter $\beta$. We do not consider the calibration of the model coefficients, as described e.g. by Meyers et al. [51], as a function of $\beta$. One reason for not employing such calibration is that it is derived for convolution filters. As we have observed in Sec. 2, this is not the case for the DG-projection and additional care is required to derive a consistent calibration procedure. Additionally it has been shown by Meyers et al. [51] that the model constant is dependent on the ratio $\Delta / \eta$, where $\eta$ is the Kolmogorov scale, when $\Delta / \eta \leqslant 100$. This is the case when the DG-LES resolution falls at the beginning of the dissipation range as considered here. Thus we also make no attempt at providing a general calibration of the model coefficient from the employed DNS/LES data as the results would be dependent on this parameter in the range of validity of the DG-VMS approach. In order to facilitate the analysis, all the results are presented with the model constant selected such that the modelled dissipation spectrum presents the same maximum value as that of the ideal SGS dissipation spectrum. The employed values of the model coefficient are reported in Table 1.

\begin{tabular}{lccc}
\hline & $\beta=0.25$ & $\beta=0.5$ & $\beta=0.75$ \\
\hline all-all & 0.094 & 0.096 & 0.098 \\
Vreman & 0.099 & 0.117 & 0.162 \\
\hline Smagorinsky & \multicolumn{3}{c}{0.090} \\
\hline
\end{tabular}

Table 1: TGV at $\operatorname{Re}=20000, p=7, k_{\mathrm{DG}}=288:$ Model coefficients selected for the Smagorinsky and DG-VMS model using the BR2 scheme $\left(\eta_{b r 2}=2\right)$.

In Fig. 16 we report the ideal and model SGS dissipation spectrum corresponding to the Smagorinsky model and the DG-VMS approach using the BR2 scheme with $\eta_{b r 2}=2$.

It is obvious from this figure that, as already shown by other authors, the Smagorinsky model provides excessive dissipation at low wavenumbers. This effect is drastically reduced by employing the considered variants of the DG-VMS approach. As expected, increasing the value of $\beta$ restricts the action of the SGS model on progressively finer scales and, for a fixed model 

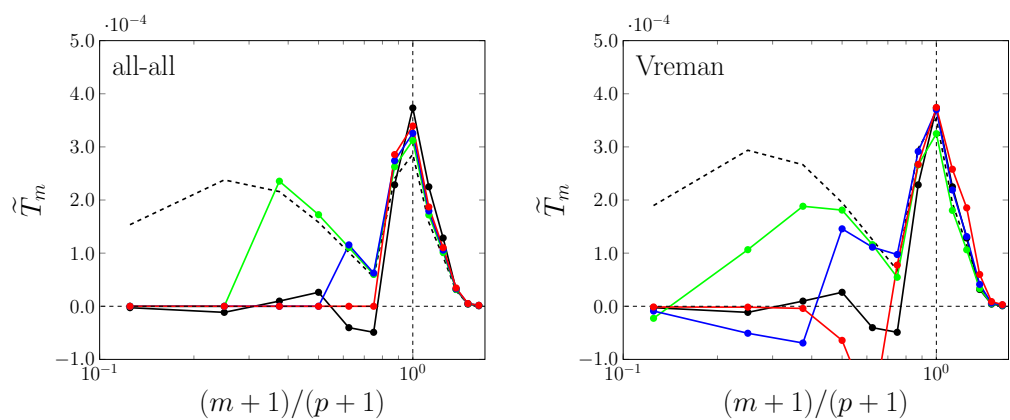

Figure 17: TGV at $\operatorname{Re}=20000, p=7, k_{\mathrm{DG}}=288:$ Ideal modal energy transfer (black solid) and modelled modal energy transfer provided by the Smagorinsky model (dashed) and two variants of the DG-VMS approach for: $\beta=0.25$ (green), $\beta=0.5$ (blue), and $\beta=0.75$ (red) using the BR2 scheme $\left(\eta_{b r 2}=2\right)$.

constant, reduces the total SGS dissipation.

In particular for $\beta=0.75$ the SGS model acts only on the range of scales $\left[k_{\mathrm{DG}} / 3, k_{\mathrm{DG}}\right]$ and provides a relatively accurate agreement with the ideal energy transfer. The quality of the agreement for wavenumbers close to and above $\tilde{k}_{\mathrm{DG}}$ was however found to be sensitive to the discretization employed for the DG-VMS model, such as the value of $\eta_{b r 2}$ or the use of the BR1 scheme rather than the BR2 scheme. The interested reader is referred to [52] for a more complete discussion on this topic.

For lower values of $\beta$ however the distribution of the modelled SGS dissipation does not correspond to the ideal SGS dissipation for any of the considered discretizations. This effect is particularly marked for $\beta<0.5$ and has also been observed by employing the DG-LES solution at the intermediate resolution $k_{\mathrm{DG}}=144$ (not reported here).

We further remark that the SGS dissipation spectrum decays smoothly for low wavenumbers for all variants of the DG-VMS approach. This is expected as the high-pass projection filter is not sharp in Fourier space. This result illustrates an advantage of using a modal decomposition as opposed to an orthogonal spectral filter as Sagaut and Levasseur [27] have shown that a smooth decay of the SGS dissipation spectrum leads to improved results in a-posteriori tests. Nonetheless, analysing Fig. 16, we observe that both VMS approaches present a negligible amount of SGS dissipation acting on the large resolved scales. This confirms that the DG-VMS approach is not able to replicate the viscous-type behaviour observed at low wavenumbers when the resolution limit falls within the inertial range.

Comparing the two variants we observe that the all-all approach presents a marginally better agreement with the ideal SGS dissipation spectrum. We recall that this variant presents a much lower computational cost for modal DG methods. On the other hand, for the Vreman approach the SGS stress is computed from the filtered gradients. This implies that the SGS stress is aligned with the small-scale gradients and tends to zero when the flow is well resolved and the solution is represented entirely by the large-scale component. An analysis of the alignment between the ideal SGS stress and the modelled one could therefore be employed in order to draw more definitive conclusions.

The conclusions drawn above are confirmed by analysing the modal energy transfer and eddy viscosity presented in Figs. 17 and 18.

It is clear from these figures that for the all-all approach a value of $\beta=0.75$ must be em- 

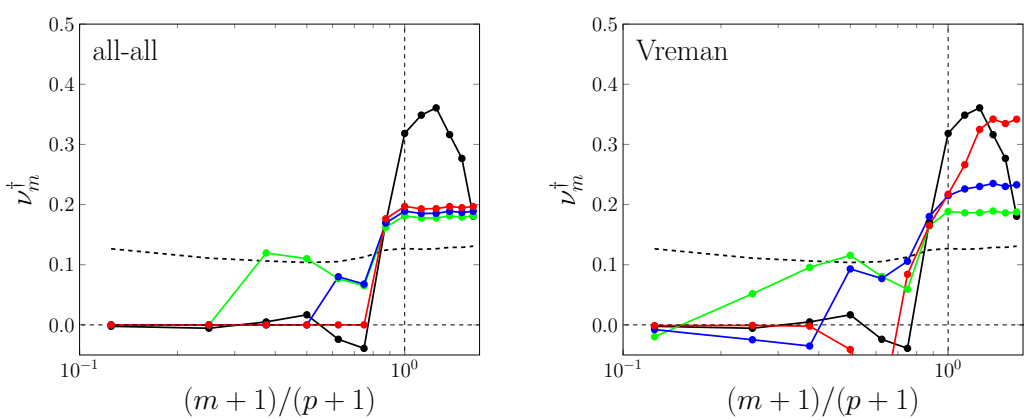

Figure 18: TGV at $\operatorname{Re}=20000, p=7, k_{\mathrm{DG}}=288:$ Ideal modal eddy viscosity (black solid) and modelled modal eddy viscosity provided by the Smagorinsky model (dashed) and two variants of the DG-VMS approach for: $\beta=0.25$ (green), $\beta=0.5$ (blue), and $\beta=0.75$ (red) using the BR2 scheme $\left(\eta_{b r 2}=2\right)$.
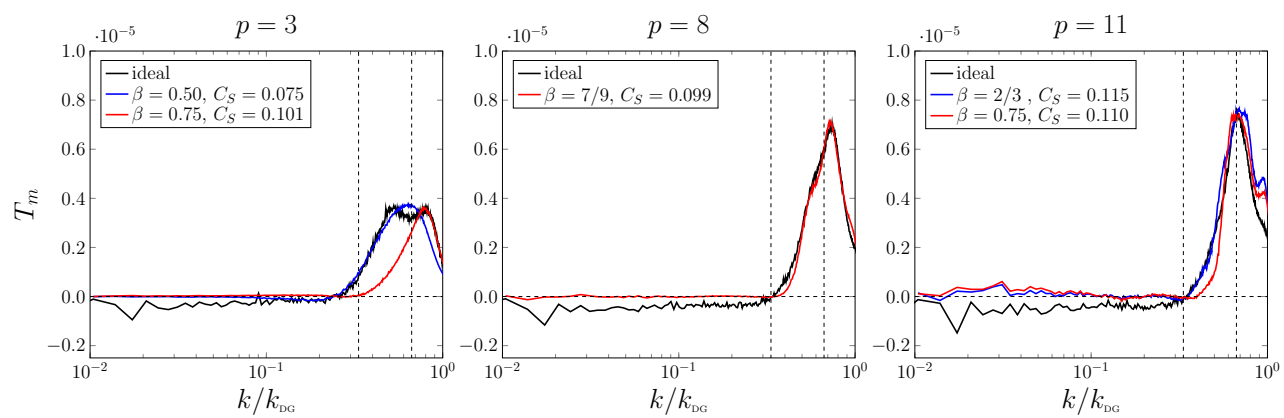

Figure 19: TGV Re $=20$ 000: Ideal SGS dissipation spectrum and model dissipation spectrum using the all-all DG-VMS approach with $C_{S}=0.1$ using the BR2 scheme for $k_{\mathrm{DG}}=288$ and $p=3$ (left), $p=8$ (center) and $p=11$ (right). The values $\tilde{k}_{\mathrm{DG}}$ and $k_{\mathrm{DG}} / 3$ are marked by dash-dotted lines.

ployed in order to mimic the negligible ideal energy transfer at low mode-numbers. In this case a remarkably good agreement is obtained with both the modal energy transfer and eddy viscosity, confirming the conclusion drawn from the spectral energy transfer analysis. A different behaviour is obtained for the Vreman model as $\widetilde{T}_{m}$ is positive for $(m+1) /(p+1)=\beta$ and becomes negative for lower values of $m$.

The presented analysis has been repeated for different values of the polynomial degree $p$. Similar conclusions have been obtained with regards to the comparison of the two variants and the sensitivity of the model dissipation spectrum to $\beta$ (not reported here). However, the optimal value of $\beta$ appears to be dependent on $p$ when $p \leq 4$. This result is expected from the a-priori analysis carried out in Sec. 5.2. To illustrate this, we report in Fig. 19 the ideal SGS dissipation spectrum and the model energy transfer for $p=3,8$ and 11 using the DG-VMS all-all approach and the BR2 scheme $\left(\eta_{b r 2}=2\right)$.

While the results obtained for $p=8$ and 11 agree with those previously presented, for $p=3$ the ideal SGS dissipation spectrum does not present a single peak at $\tilde{k}_{\mathrm{DG}}$. In this case the ideal SGS dissipation is in between that provided by the DG-VMS model for $\beta=0.5$ and $\beta=0.75$. This result indicates that for $p \leq 4$ the DG-VMS approach might not be able to replicate the distribution of the ideal SGS dissipation. 
The analysis presented up to this point has focused on the ability of the DG-VMS approach to replicate the ideal energy transfer mechanism when the SGS dissipation acting on large-resolved scales is negligible.

We have observed in Sec. 5 that when the resolution limit falls within the inertial range a viscous-type behaviour can be observed and is dominant at wavenumbers below $k_{\mathrm{DG}} / 3$. It appears from these analyses that the DG-VMS approach is not able to replicate this mechanism.

Modifying or adapting $\beta$ does not lead to a better representation of the SGS dissipation spectrum. However this parameter allows us to control the set of scales on which the SGS dissipation acts and the total amount of SGS dissipation. Thus the DG-VMS approach can nonetheless lead to improved results with respect to the standard or dynamic Smagorinsky model which might introduce excessive dissipation on the large resolved scales. This also explains the promising results observed for the local VMS approach proposed by Ramakrishnan and Collis [26] and for the dynamic partition selection algorithm for the DG-VMS approach proposed by Naddei et al. [53].

In the context of high Reynolds numbers and typical LES resolutions, the limitations of the DG-VMS model could be mitigated by combining it with an eddy-viscosity model, which mimics the viscous-type behaviour observed at low wavenumbers. The ideal SGS dissipation spectrum could be therefore approximated by employing a mixed Smagorinsky+DG-VMS model where the Smagorinsky model acts on all scales and the VMS approach with $\beta=0.75$ replicates the hyperviscous behaviour dominant on wavenumbers $k>k_{\mathrm{DG}} / 3$. Similar approaches have already been proposed in the literature, e. $g$. the Smagorinsky and residual-based-VMS approach by Wang and Oberai [54], or the enhanced field model and the mixed Smagorinsky-hyperviscosity model by Jeanmart and Winckelmans [12]. Two model coefficients appear however in the resulting model. These coefficients should be dynamically adapted, e.g. using a Germano-type procedure similarly to that used in [12]. An alternative approach could consist in evaluating dynamically one of the coefficients and calibrating their ratio based on the resolution available, e.g. as a function of $h / \eta$.

In order to illustrate this idea, in Fig. 20 we consider the ideal SGS dissipation spectrum for a snapshot at $t=14$ of the TGV at $\operatorname{Re}=20000, p=7$ and $288^{3}$ dofs. In the left panel, the ideal SGS dissipation spectrum is compared to the two variants of the mixed model with constant coefficients. This figure illustrates that a dynamic mixed model even with global model coefficients has the potential, at least a-priori, to an accurate agreement with the ideal SGSdissipation. This is not the case for the standard Smagorinsky and the DG-VMS models as shown on the right panel of Fig. 20. However, the appropriateness of such a model can only be confirmed via a-posteriori testing. This is out of the scope of this research and is thus left for future work.

\section{Conclusions}

In this work we have proposed a framework for the a-priori analysis of DG-LES methods based on DNS databases. It is an extension of the classical framework for the analysis of the energy transfer between resolved and unresolved scales of Kraichnan [7] and Domaradzki et al. [8]. The proposed framework is consistent with the employed discretization and as such, allows the evaluation of the ideal SGS dissipation spectrum that needs to be modelled including the effect of discontinuities inherently present in the DG method and the particular choice of the numerical flux. 

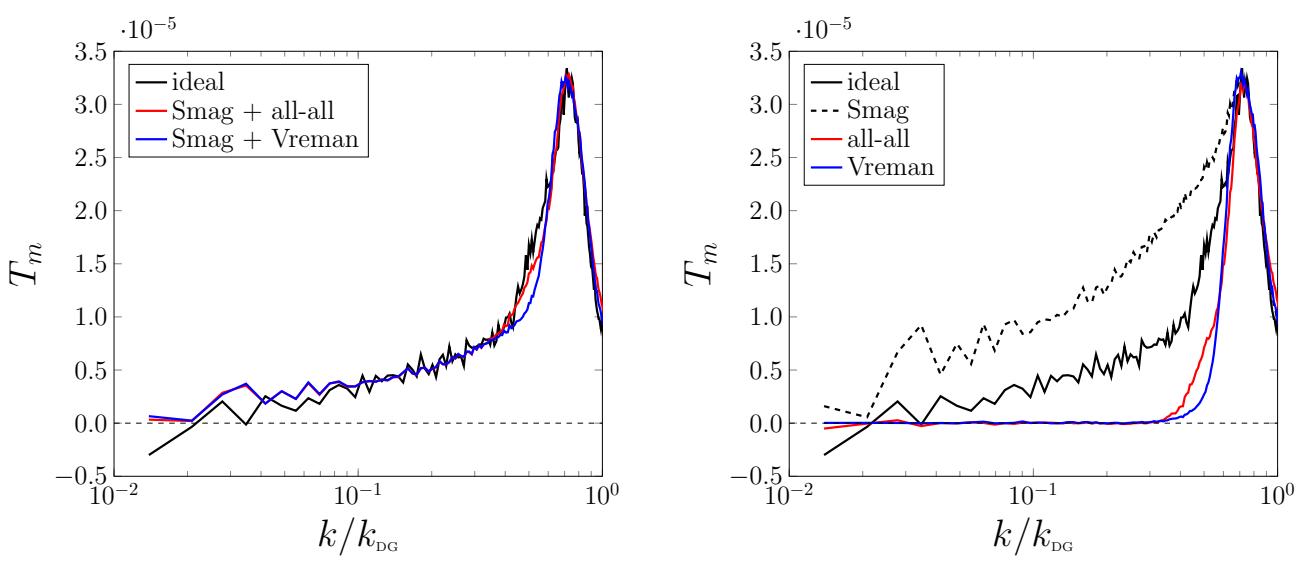

Figure 20: $\mathrm{TGV} \operatorname{Re}=20000, p=7, k_{\mathrm{DG}}=144$ : Ideal SGS dissipation spectrum and modelled dissipation spectrum for mixed Smagorinsky+DG-VMS models (left) and the Smagorinsky and standard DG-VMS models with $\beta=0.75$ (right) The model coefficients for the mixed versions are set to $C_{s, s m a g}=0.067$ and $C_{s, a-a}=0.063$ for the all-all variant and $C_{s, \text { vrem }}=0.103$ for the Vreman variant. For the standard version, the model coefficients are set to $C_{s, s m a g}=0.091$, $C_{s, a-a}=0.085$ and $C_{s, v r e m}=0.114$ respectively.

We have further introduced the concept of a modal energy transfer and eddy viscosity. These quantities can be employed to analyse a-priori LES models that rely on modifying the amount of SGS dissipation acting on different modes of the solution such as the DG-VMS model and the spectral vanishing viscosity approach.

The developed framework has been applied to a DNS database of the TGV at $\operatorname{Re}=5000$, 20000 and 40000 [23]. It has been shown that the ideal SGS dissipation spectrum presents in general a mixed viscous-hyperviscous behaviour. The viscous behaviour is dominant for wavenumbers below $\tilde{k}_{\mathrm{DG}} / 2=k_{\mathrm{DG}} / 3=n_{e l}(p+1) / 6$ whereas the hyperviscous behaviour is dominant at higher wavenumbers with a peak at $\tilde{k}_{\mathrm{DG}}$. Only when the DG resolution limit falls at the beginning of the dissipation range the energy transfer between large-resolved and unresolved scales is negligible. This corresponds to a negligible energy transfer to DG modes of modenumber $m+1 \leq 0.75(p+1)$. Under these conditions, the DG-VMS approach can provide an accurate approximation of the SGS dissipation spectrum.

A-priori analyses of the Vreman [1] and all-all [2] variants of the DG-VMS approach have been carried out. It has been shown that, when the ideal SGS dissipation acting on the large resolved scales is negligible, the best results for the DG-VMS approach are obtained for a scale-fraction parameter of $\beta=0.75$. Both variants provide a good agreement with the ideal SGS dissipation spectrum. In particular, the all-all approach more closely replicates the ideal modal energy transfer and presents a reduced computational cost.

All results have been shown to only mildly depend on the polynomial degree for $p \geq 5$ and more marked differences are observed for lower values of $p$ which lead to different optimal values of $\beta$. We therefore suggest to employ a value of $p \geq 5$ as it leads to a more consistent behaviour of the ideal energy transfer mechanism and a good agreement of the DG-VMS approach with the ideal quantities.

Finally, we have shown that the DG-VMS approach is not able to reproduce the viscous-type behaviour observed at relatively low wavenumbers when the resolution limit falls within the inertial range. Under these conditions, the a-priori analyses indicate that a mixed model based 
on the Smagorinsky model and the DG-VMS approach with $\beta=0.75$ could provide an improved agreement over a wide range of resolutions provided that the model coefficients are dynamically adapted.

\section{Acknowledgments}

This project has received funding from the European Union's Horizon 2020 research and innovation program under the Marie Skłodowska-Curie grant agreement No 675008. Part of this research was conducted during the 2018 Center for Turbulence Research (CTR) Summer Program at Stanford University. The second author would like to acknowledge funding from CTR for participation in the Summer Program. This work was performed using HPC resources from CINES and TGCC (GENCI Grants 2017-A0022A10129, 2017-A0032A07624 and 2018A0052A07624).

\section{Appendix A. The subfilter and subgrid contributions to the total LES residual}

Following an approach similar to $[40,39]$ the total DG-LES residual Eq. (7) can be rewritten in the form

$$
\begin{aligned}
\left.\mathcal{R}\left(\mathbf{u}, \overline{\mathbf{u}}_{h}, \phi\right)\right) & =\sum_{K}\left[\int_{K}\left(\mathcal{F}_{c}\left(\overline{\mathbf{u}}_{h}\right)-\mathcal{F}_{c}(\overline{\mathbf{u}})\right) \cdot \boldsymbol{\nabla} \phi \mathrm{d} \mathbf{x}-\int_{\partial K}\left(\mathbf{h}_{c}\left(\overline{\mathbf{u}}_{h}^{+}, \overline{\mathbf{u}}_{h}^{-}, \mathbf{n}^{+}\right)-\mathcal{F}_{c}(\overline{\mathbf{u}}) \cdot \mathbf{n}^{+}\right) \phi^{+} \mathrm{d} \sigma\right] \\
& +\sum_{K}\left[\int_{K}\left(\mathcal{F}_{c}(\overline{\mathbf{u}})-\overline{\mathcal{F}}_{c}(\mathbf{u})\right) \cdot \boldsymbol{\nabla} \phi \mathrm{d} \mathbf{x}-\int_{\partial K}\left(\mathcal{F}_{c}(\overline{\mathbf{u}})-\overline{\mathcal{F}}_{c}(\mathbf{u})\right) \cdot \mathbf{n}^{+} \phi^{+} \mathrm{d} \sigma\right]
\end{aligned}
$$

Using integration by parts and summing over all elements for the integrals that do not depend on $\overline{\mathbf{u}}_{h}$ we obtain:

$$
\begin{aligned}
\left.\mathcal{R}\left(\mathbf{u}, \overline{\mathbf{u}}_{h}, \phi\right)\right)=\sum_{K} & {\left[\int_{K}\left(\mathcal{F}_{c}\left(\overline{\mathbf{u}}_{h}\right)-\mathcal{F}_{c}(\overline{\mathbf{u}})\right) \cdot \nabla \phi \mathrm{d} \mathbf{x}-\int_{\partial K}\left(\mathbf{h}_{c}\left(\overline{\mathbf{u}}_{h}^{+}, \overline{\mathbf{u}}_{h}^{-}, \mathbf{n}^{+}\right)-\mathcal{F}_{c}(\overline{\mathbf{u}}) \cdot \mathbf{n}^{+}\right) \phi^{+} \mathrm{d} \sigma\right] } \\
& +\int_{\Omega_{h}} \boldsymbol{\nabla}\left(\overline{\mathcal{F}}_{c}(\mathbf{u})-\mathcal{F}_{c}(\overline{\mathbf{u}})\right) \phi \mathrm{d} \mathbf{x}
\end{aligned}
$$

Employing the same procedure presented in Sec. 3 the contribution of the unresolved scales to the evolution of the DG-LES solution Eq. (13) can be expressed as

$$
\begin{aligned}
\mathrm{R}\left(\mathbf{u}, \overline{\mathbf{u}}_{h}\right) & =\overbrace{\sum_{i} \sum_{K}\left[\int_{K}\left(\mathcal{F}_{c}\left(\overline{\mathbf{u}}_{h}\right)-\mathcal{F}_{c}(\overline{\mathbf{u}})\right) \cdot \boldsymbol{\nabla} \psi_{K}^{i} \mathrm{~d} \mathbf{x}-\int_{\partial K}\left(\mathbf{h}_{c}\left(\overline{\mathbf{u}}_{h}^{+}, \overline{\mathbf{u}}_{h}^{-}, \mathbf{n}^{+}\right)-\mathcal{F}_{c}(\overline{\mathbf{u}}) \cdot \mathbf{n}^{+}\right) \psi_{K}^{i,+} \mathrm{d} \sigma\right] \psi_{K}^{i}}^{\mathrm{R}_{S G S}\left(\overline{\mathbf{u}}, \overline{\mathbf{u}}_{h}\right)} \\
& +\underbrace{\sum_{i} \sum_{K} \int_{\Omega_{h}} \boldsymbol{\nabla} \cdot\left(\overline{\mathcal{F}}_{c}(\mathbf{u})-\mathcal{F}_{c}(\overline{\mathbf{u}})\right) \psi_{K}^{i} \mathrm{~d} \mathbf{x} \psi_{K}^{i}}_{\mathrm{R}_{S F S}(\mathbf{u}, \overline{\mathbf{u}})},
\end{aligned}
$$


where the second term can be rewritten as

$$
\mathrm{R}_{S F S}(\mathbf{u}, \overline{\mathbf{u}})=\mathbb{P}_{S_{h}^{p}}\left[\boldsymbol{\nabla} \cdot\left(\overline{\mathcal{F}}_{c}(\mathbf{u})-\mathcal{F}_{c}(\overline{\mathbf{u}})\right)\right] .
$$

The total influence of the unresolved scales on the evolution of the DG-LES solution can be therefore expressed as the combination of two contributions. The former is a subgrid-scale residual term $\mathrm{R}_{S G S}$, which depends only on the DG-LES solution $\overline{\mathbf{u}}_{h}$ and the subgrid scales $\overline{\mathbf{u}}-\overline{\mathbf{u}}_{h}$. The latter is a subfilter-scale residual term $\mathrm{R}_{S F S}$, which corresponds to the projection on the DGLES space of the divergence of the subfilter stresses $\overline{\mathcal{F}}_{c}(\mathbf{u})-\mathcal{F}_{c}(\overline{\mathbf{u}})$ which depend only on the spatially filtered solution $\overline{\mathbf{u}}$ and the subfilter scales $\mathbf{u}-\overline{\mathbf{u}}$.

We remark that in this work, in contrast to $e . g$. [39] or [40], we only assume that the filter $\overline{(\cdot)}$ commutes with spatial derivatives and do not make any further assumption about its regularity. Such properties are however fundamental for the mathematical analysis of subfilter-scale stresses and the derivation of various LES models such as the Leonard [55] or the Bardina [56] models.

\section{Appendix B. Energy and dissipation spectra computation}

For the computation of the energy and dissipation spectra we need to evaluate the Fourier transform of the velocity field, SGS residual, and SGS model term. For this purpose we employ the Fast Fourier Transform (FFT) algorithm as implemented in the FFTW-3.3.8 library [57].

The FFT algorithm requires the solution to be known on an uniform Cartesian grid. The variable of which we want to compute the FFT is therefore sampled on a post-processing grid formed by the union of $n_{e l}^{3}$ uniform Cartesian grids centered on each cell and composed of $q_{s}^{3}$ sampling points. The post-processing grid on the domain $[-\pi, \pi]^{3}$ is thus composed of the Cartesian product of the coordinates $\left(j-\frac{1}{2}\right) \frac{2 \pi}{q_{s} n_{e l}}$ for $j=1, \ldots, N_{F F T}=q_{s} n_{e l}$. The value of $q_{s}$ must be sufficiently large to evaluate accurately the Fourier coefficients corresponding to the wavenumbers of interest (that is at least up to $k_{\mathrm{DG}}$ ).

Indeed, the presence of discontinuities in the DG-LES field leads to a reduction in the order of convergence of the FFT algorithm. We remark that the FFT algorithm relies on the trapezoidal integration rule which presents an order of accuracy $O\left(N_{F F T}{ }^{-1}\right)$ in the presence of discontinuities, as opposed to the exponential convergence obtained for smooth functions. In this case the use of $n_{e l}(p+1)$ points per direction, as is usually found in the literature of DG-LES, is not sufficient for the evaluation of the energy and dissipation spectra. For this reason, we employ at least $3 n_{e l}(p+1)$ points per direction and verify that increasing this value does not modify the energy and dissipation spectra at wavenumbers below $k_{\mathrm{DG}}$.

In order to illustrate the need for a sufficiently high number of points for the evaluation of the FFT, we report in Fig. B.21 the energy spectra of the DG-LES solution of the TGV configuration at $\operatorname{Re}=20000$ and $t=14$ using $p=5$ and 7 and a total of 288 degrees of freedom for various values of $q_{s}$. It can be observed that the FFT is inaccurate for low values of $q_{s}$ even at wavenumbers below $k_{\mathrm{DG}}$. Slight differences can be observed for wavenumbers below $\tilde{k}_{\mathrm{DG}}$ and in some cases marked differences can appear at wavenumbers close to $k_{\mathrm{DG}}$. This is visible from the bump in the energy spectrum at $k_{\mathrm{DG}}$ for $q_{s}=p+1$ on the right panel of Fig. B.21 corresponding to $p=7$.

Nonetheless, the spectrum converges as $q_{s}$ is increased and a value of $q_{s} \approx 3 n_{e l}(p+1)$ appears sufficient to obtain the Fourier transform for wavenumbers up to $k_{\mathrm{DG}}$. Further increasing the value of $q_{s}$ leads to the slow convergence of the tail of the spectrum associated with the DG-LES discontinuities. 

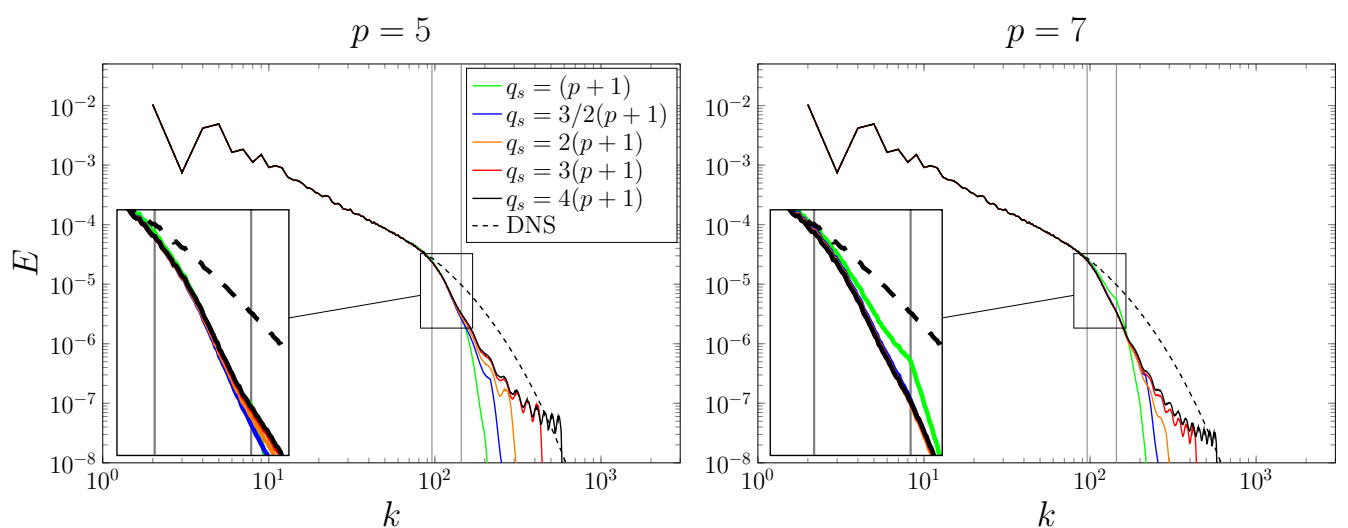

Figure B.21: $\mathrm{TGV}$ at $\operatorname{Re}=20000, t=14, k_{\mathrm{DG}}=144$ : Energy spectrum for $p=5$ (left) and $p=7$ (right) for various values of $q_{s}$.

We note that alternative techniques can be employed to evaluate the Fourier transform avoiding the use of a large number of post-processing points. Such methodologies include the Non Uniform Fast Fourier Transform (NUFFT) $[58,59]$ and the Conformal Fourier Transform (CFT) [60].

\section{Appendix C. Sensitivity of the ideal energy transfer to the DG-LES filter}

As discussed in Sec. 2, all the results presented have been obtained by defining the ideal DG-LES solution as the $L^{2}$-projection on the discretization space of the DNS solution filtered with a sharp spectral filter removing wavenumbers $\|\mathbf{k}\|_{\infty} \geq k_{\mathrm{DG}}$. Other possible definitions can be considered. Among them, the $L^{2}$-projection of the DNS solution on the DG discretization space is an interesting candidate.

The effect of these two different definitions on the results obtained is therefore investigated. For this purpose we employ the snapshot at $t=14$ of the TGV at $\operatorname{Re}=20000$. In order to simplify the notation we will refer to the $L^{2}$-projection of the DNS solution on the DG space as simply the DG-projection in contrast to the employed definition of ideal DG-LES solution.

In Fig. C.22 we report the energy spectra corresponding to the ideal DG-LES and the DGprojection for three resolutions. We observe that for all resolutions the energy spectra are indistinguishable for wavenumbers up to $\tilde{k}_{\mathrm{DG}}$. Moreover we notice higher values of the tail of the energy spectra for the DG-projection which is especially evident for the coarsest resolution (left panel of Fig. C.22). This indicates, as one would expect, that the $L^{2}$-projection of the DNS field presents stronger discontinuities than the $L^{2}$-projection of the filtered field. Nonetheless, $\tilde{k}_{\mathrm{DG}}$ appears to be a relevant wavenumber identifying the resolution properties of DG using both definitions.

In Figs. C. 23 and C.24 we present the spectral and modal energy transfer for the same resolutions. These figures illustrate a fair agreement between results obtained with the two definitions. The most remarkable differences appear in Fig. C.23 for the relatively low wavenumbers. Indeed the DG-projection leads to a more erratic behaviour of the spectral energy transfer which could be explained by the presence of aliasing errors as described in Sec. 2. 

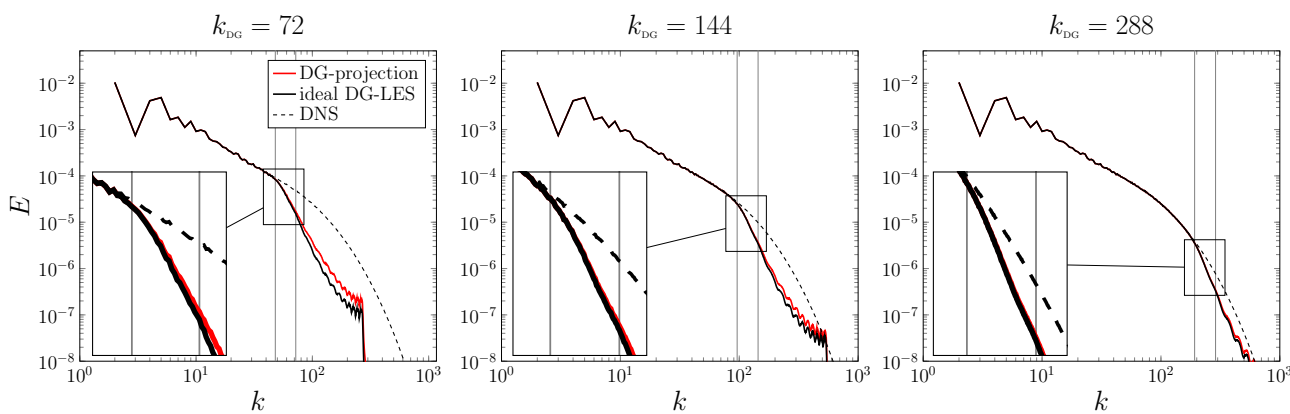

Figure C.22: TGV at Re $=20000$ : Energy spectra of the DNS data, the ideal DG-LES solution, and DG-projection for three resolutions with $p=7$. Close-up view for wavenumbers between $\tilde{k}_{\mathrm{DG}}$ and $k_{\mathrm{DG}}$.
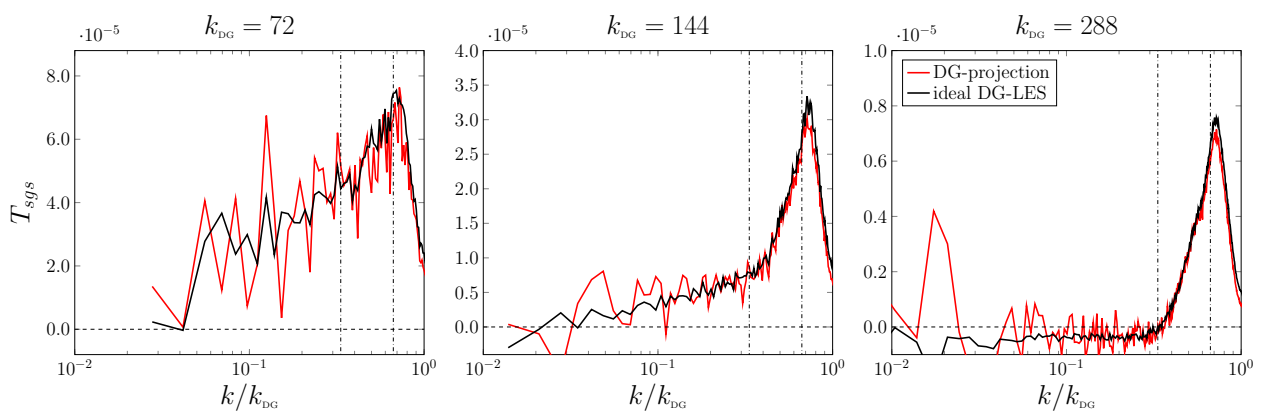

Figure C.23: TGV at $\mathrm{Re}=20$ 000: Ideal SGS dissipation spectrum of the ideal DG-LES solution and the DG-projection for three discretizations with $p=7$. Dashed lines mark values of $\tilde{k}_{\mathrm{DG}}$ and $k_{\mathrm{DG}} / 3$.

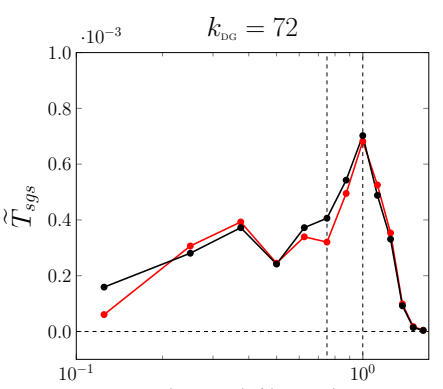

$(m+1) /(p+1)$

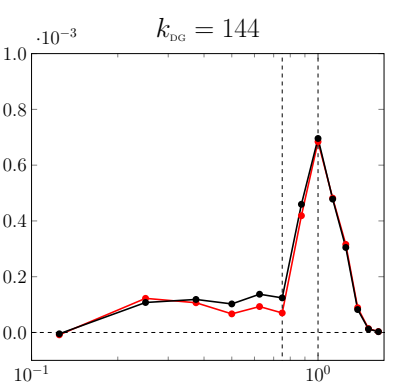

$(m+1) /(p+1)$

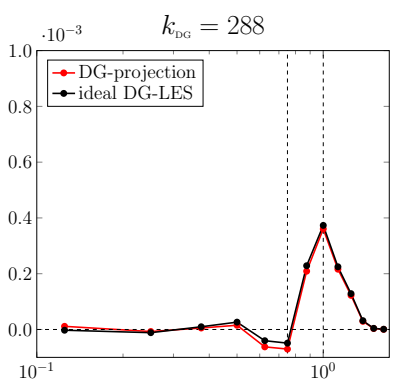

$(m+1) /(p+1)$

Figure C.24: TGV at Re = 20 000: Ideal modal energy transfer of the ideal DG-LES solution and the DG-projection for three discretizations with $p=7$. Dashed lines indicate mode-numbers $m+1=0.75(p+1)$ and $m=p$. 

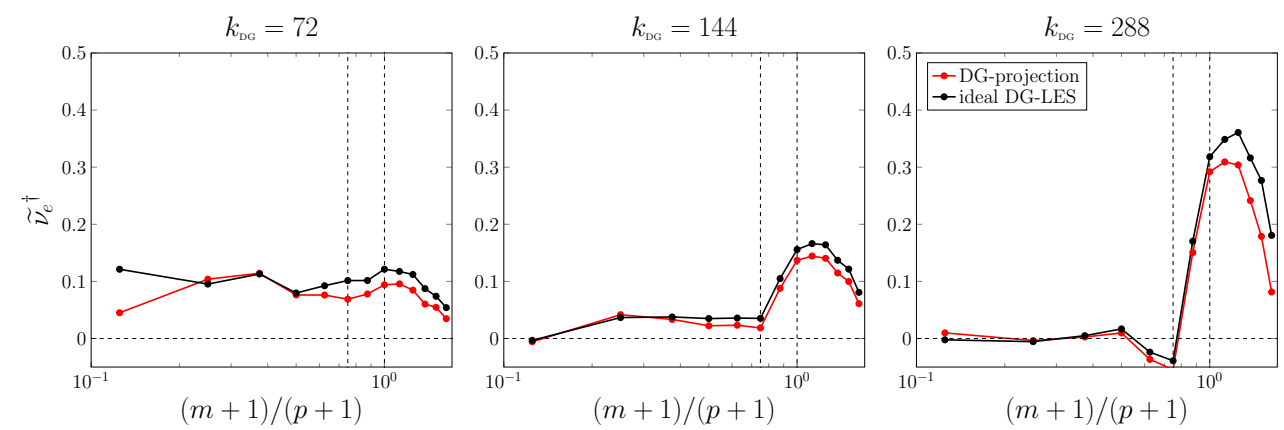

Figure C.25: TGV at Re $=20000$ : Ideal modal eddy viscosity of the ideal DG-LES solution and the DG-projection for three discretizations with $p=7$. Dashed lines indicate mode-numbers $m+1=0.75(p+1)$ and $m=p$.

Slightly more significant differences can be observed in Fig. C.25 which reports the modal eddy viscosity employing the BR1 scheme. In this figure we can identify lower values of the modal eddy viscosity at relatively high mode-numbers. These can be explained by the presence of stronger discontinuities and higher values of the lifting coefficients used for the BR1 scheme and therefore, higher values of the diffusive term in the denominator of Eq. (22) at high modenumbers.

Overall the results obtained demonstrate that, with the exception of small differences, the definition of the reference DG-LES solution as the $L^{2}$-projection of the DNS field leads to the same conclusions drawn by employing the current definition of the ideal DG-LES solution.

\section{Appendix D. Choice of the large-scale space}

In Sec. 3 we have defined the space $W_{h}^{m}=\operatorname{span}\left\{\psi_{K}^{\mathbf{m}}, \forall K \in \Omega_{h}, m-\frac{1}{2}<\|\mathbf{m}\| \leq m+\frac{1}{2}\right\}$. With this choice the assumption of $\tilde{T}_{s g s}(m)=0$ for $m \leq p_{L}$ corresponds to the VMS approach defining the large-scale space as $V^{L}:=\bigcup_{m \leq p_{L}} W_{h}^{m}$ as described in Sec. 4. Other definitions are possible, in particular the most common choice is to define $V^{L}:=S_{h}^{p_{L}}$ which corresponds to assuming that $\tilde{T}^{c}(m)=0$ for $m \leq p_{L}$ where

$$
\widetilde{T}^{c}(m):=\mathcal{R}\left(\mathbf{u}, \overline{\mathbf{u}}_{h}, \mathbb{P}_{W_{h}^{m, c}}\left[\overline{\mathbf{u}}_{h}\right]\right),
$$

with $W_{h}^{m, c}=\operatorname{span}\left\{\psi_{K}^{\mathbf{m}}, \forall K \in \Omega_{h},\|\mathbf{m}\|_{\infty}=m\right\}$. It is immediate to show that $W_{h}^{m, c} \equiv S_{h}^{m} \backslash S_{h}^{m-1}$ for $m>0$ and that $W_{h}^{0, c} \equiv S_{h}^{0}$.

The definition employed throughout this work Eq. (20) corresponds to analysing the modal energy transfer by grouping together modes over spherical shells characterized by $m-\frac{1}{2}<$ $\|\mathbf{m}\| \leq m+\frac{1}{2}$, whereas Eq. (D.1) corresponds to grouping modes over cubic shells characterized by $\|\mathbf{m}\|_{\infty}=m$.

We argue that Eq. (20) allows for a more consistent description of the modal energy transfer mechanism. To justify this choice we report in Figs. D.26 and D.27 the contour plots of the modal eddy viscosity $\widetilde{v}(\mathbf{m})$ for $p=7$ and $p=11$ defined as 

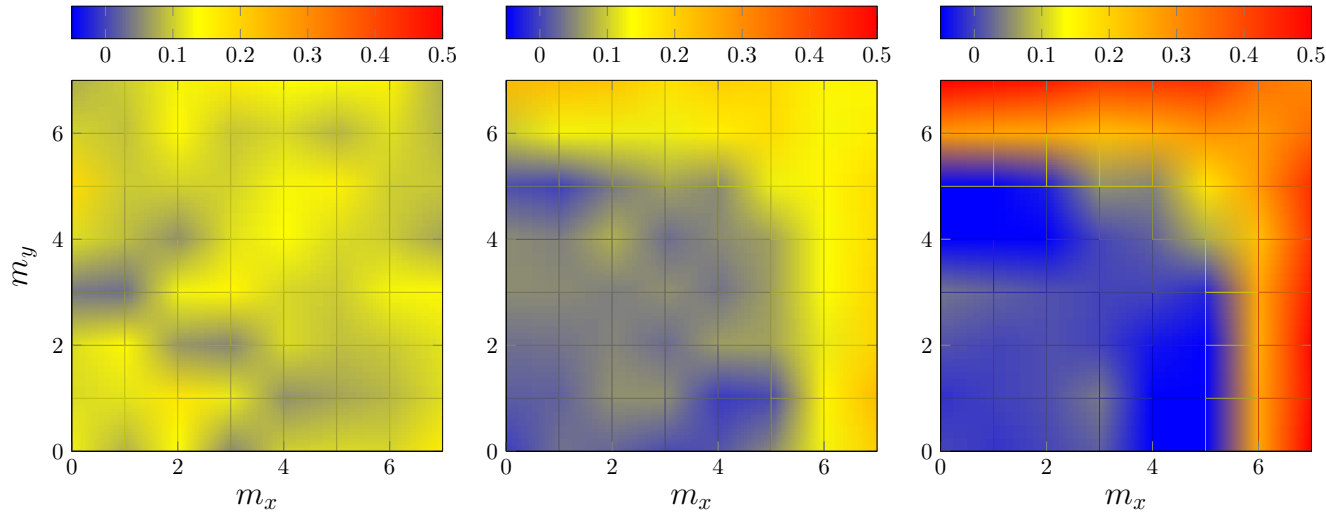

Figure D.26: TGV at $\operatorname{Re}=20,000, t=14$ : Contour plot of $\widetilde{v}^{\dagger}(\mathbf{m})$ at constant $m_{z}=0$ for $p=7$ and $144^{3}, 288^{3}$ and $576^{3}$ dofs (left to right) using the BR1 scheme.
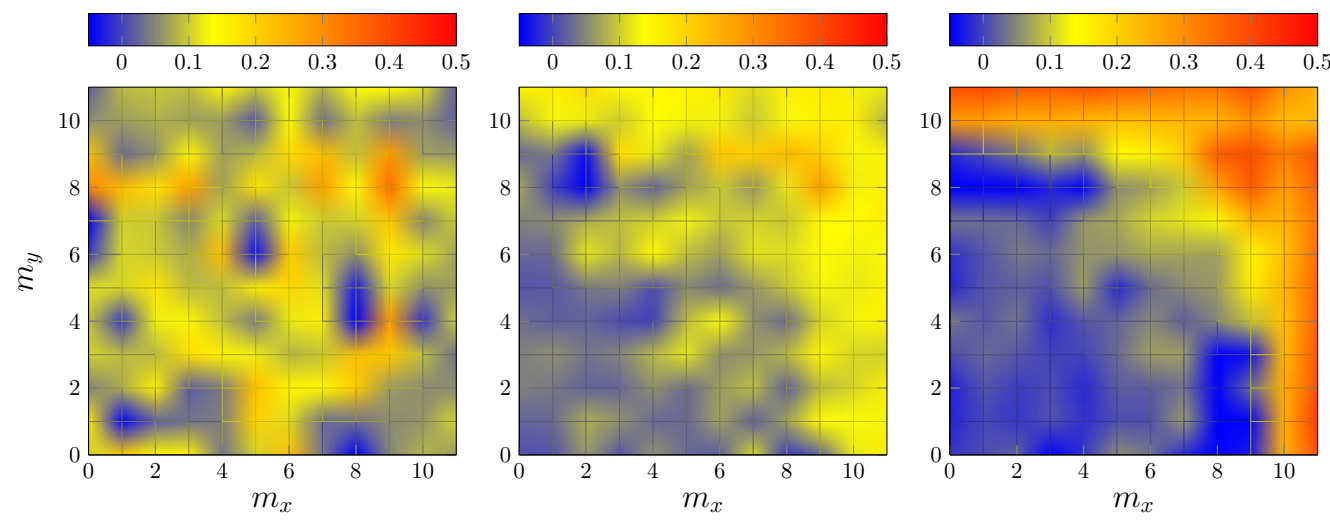

Figure D.27: TGV at $\operatorname{Re}=20,000, t=14$ : Contour plot of $\widetilde{v}^{\dagger}(\mathbf{m})$ at constant $m_{z}=0$ for $p=11$ and $144^{3}, 288^{3}$ and $576^{3}$ dofs (left to right) using the BR1 scheme.

$$
\widetilde{v}(\mathbf{m}):=\frac{\sum_{K \in \Omega_{h}}{\widetilde{\overline{\mathbf{u}}_{h}}}^{\mathbf{m}, K} \cdot \mathcal{R}\left(\mathbf{u}, \overline{\mathbf{u}}_{h}, \psi_{K}^{\mathbf{m}}\right)}{\sum_{K \in \Omega_{h}} \widetilde{\mathbf{u}}_{h}^{\mathbf{m}, K} \cdot \mathcal{L}_{v}\left(\overline{\mathbf{u}}_{h}, \psi_{K}^{\mathbf{m}}\right)} .
$$

and normalized as in Eq. (23).

We observe that the isolevel curves for $\widetilde{v}^{\dagger}(\mathbf{m})$ are better approximated by spheres (circles in the plot) rather than by cubes centred in $(0,0,0)$. Therefore we assume that improved results can be obtained for LES models by modifying the modal eddy viscosity as a function of $\|\mathbf{m}\|$ rather than $\|\mathbf{m}\|_{\infty}$.

\section{References}

[1] A. Vreman, The filtering analog of the variational multiscale method in large-eddy simulation, Phys Fluids 15 (8) (2003) L61-L64. 
[2] J.-B. Chapelier, M. de la Llave Plata, E. Lamballais, Development of a multiscale LES model in the context of a modal discontinuous Galerkin method, Comput Method Appl M 307 (2016) 275-299.

[3] R. S. Rogallo, P. Moin, Numerical simulation of turbulent flows, Annu Rev Fluid Mech 16 (1) (1984) 99-137.

[4] M. Lesieur, O. Métais, P. Comte, Large-eddy Simulations of Turbulence, Cambridge University Press, 2005.

[5] P. Sagaut, Large eddy simulation for incompressible flows: an introduction, Springer Science \& Business Media, 2006.

[6] W. Heisenberg, On the theory of statistical and isotropic turbulence, P Roy Soc Lond A Mat 195 (1042) (1948) $402-406$.

[7] R. H. Kraichnan, Eddy viscosity in two and three dimensions, J Atmos Sci 33 (1976) 1521-1536.

[8] J. A. Domaradzki, W. Liu, M. E. Brachet, An analysis of subgrid-scale interactions in numerically simulated isotropic turbulence, Phys Fluids A 5 (1993) 1747-1759.

[9] D. McComb, A. Young, Explict-scales projections of the partitioned non-linear term in direct numerical simulation of the Navier-Stokes equation, in: Second Monte Verita Colloquium on Fundamental Problematic Issues in Fluid Turbulence, 1998.

[10] O. Métais, M. Lesieur, Spectral large-eddy simulation of isotropic and stably stratified turbulence, J Fluid Mech 239 (1992) 157-194.

[11] J. Smagorinsky, General circulation experiments with the primitive equations: I. the basic experiment, Mon Weather Rev 91 (3) (1963) 99-164.

[12] H. Jeanmart, G. Winckelmans, Investigation of eddy-viscosity models modified using discrete filters: A simplified "regularized variational multiscale model" and an "enhanced field model", Phys Fluids 19 (5) (2007) 055110.

[13] M. Germano, U. Piomelli, P. Moin, W. H. Cabot, A dynamic subgrid-scale eddy viscosity model, Phys Fluids A: Fluid 3 (7) (1991) 1760-1765.

[14] T. J. R. Hughes, G. N. Wells, A. A. Wray, Energy transfers and spectral eddy viscosity in large-eddy simulations of homogeneous isotropic turbulence: Comparison of dynamic Smagorinsky and multiscale models over a range of discretizations, Phys Fluids 16 (11) (2004) 4044-4052.

[15] T. J. R. Hughes, L. Mazzei, K. E. Jansen, Large eddy simulation and the variational multiscale method, Comput Visualization Sci 3 (2000) 47-59.

[16] V. Gravemeier, The variational multiscale method for laminar and turbulent flow, Arch Comput Method E 13 (2) (2006) 249.

[17] N. Ahmed, T. Chacón Rebollo, V. John, S. Rubino, A review of Variational Multiscale Methods for the simulation of turbulent incompressible flows, Arch Comput Method E 24 (2017) 115-164.

[18] U. Rasthofer, V. Gravemeier, Recent developments in Variational Multiscale Methods for Large-Eddy Simulation of turbulent flow, Arch Comput Method E (2017) 1-44.

[19] S. S. Collis, Monitoring unresolved scales in multiscale turbulence modeling, Phys Fluids 13 (6) (2001) $1800-1806$.

[20] B. Cockburn, C.-W. Shu, Runge-Kutta Discontinuous Galerkin Methods for Convection-Dominated Problems, J Sci Comput 16 (3) (2001) 173-261. doi:10.1023/A:1012873910884. URL https : //doi .org/10.1023/A : 1012873910884

[21] D. Leslie, G. Quarini, The application of turbulence theory to the formulation of subgrid modelling procedures, $\mathrm{J}$ Fluid Mech 91 (1) (1979) 65-91.

[22] S. Cerutti, C. Meneveau, O. M. Knio, Spectral and hyper eddy viscosity in high-Reynolds-number turbulence, J Fluid Mech 421 (2000) 307-338.

[23] E. Lamballais, T. Dairay, S. Laizet, J. Vassilicos, Implicit/Explicit Spectral Viscosity and Large-Scale SGS Effects, in: Direct and Large-Eddy Simulation XI, Springer, 2019, pp. 107-113.

[24] T. J. Hughes, L. Mazzei, A. A. Oberai, A. A. Wray, The multiscale formulation of large eddy simulation: Decay of homogeneous isotropic turbulence, Phys Fluids 13 (2) (2001) 505-512.

[25] J. Holmen, T. J. Hughes, A. A. Oberai, G. N. Wells, Sensitivity of the scale partition for variational multiscale large-eddy simulation of channel flow, Phys Fluids 16 (3) (2004) 824-827.

[26] S. Ramakrishnan, S. S. Collis, Partition selection in multiscale turbulence modeling, Phys Fluids 18 (2006) 075105.

[27] P. Sagaut, V. Levasseur, Sensitivity of spectral variational multiscale methods for large-eddy simulation of isotropic turbulence, Phys Fluids 17 (2005) 035113.

[28] J. Meyers, P. Sagaut, Evaluation of smagorinsky variants in large-eddy simulations of wall-resolved plane channel flows, Phys Fluids 19 (9) (2007) 095105.

[29] D. K. Lilly, The representation of small-scale turbulence in numerical simulation experiments, in: IBM Scientific Computing Symposium on Environmental Sciences, 1966.

[30] J. Meyers, P. Sagaut, On the model coefficients for the standard and the variational multi-scale Smagorinsky model, J Fluid Mech 569 (2006) 287-319.

[31] R. Cocle, L. Bricteux, G. Winckelmans, Scale dependence and asymptotic very high reynolds number spectral behavior of multiscale subgrid models, Phys Fluids 21 (8) (2009) 085101.

[32] L. Bricteux, M. Duponcheel, G. Winckelmans, A multiscale subgrid model for both free vortex flows and wall- 
bounded flows, Phys Fluids 21 (10) (2009) 105102.

[33] S. Pope, Large-eddy simulation using projection onto local basis functions, in: Lect Notes Phys, Springer, 2001, pp. 239-265.

[34] A. Vreman, The adjoint filter operator in large-eddy simulation of turbulent flow, Phys Fluids 16 (6) (2004) $2012-$ 2022.

[35] S. Collis, The DG/VMS method for unified turbulence simulation, in: 32nd AIAA Fluid Dynamics Conference and Exhibit, 2002, p. 3124.

[36] A. D. Beck, D. G. Flad, C.-D. Munz, Neural networks for data-based turbulence models, arXiv preprint arXiv: $1806.04482(2018)$.

[37] F. van der Bos, B. J. Geurts, Computational error-analysis of a discontinuous galerkin discretization applied to large-eddy simulation of homogeneous turbulence, Comput Method Appl M 199 (13-16) (2010) 903-915.

[38] D. Gottlieb, S. A. Orszag, Numerical analysis of spectral methods: theory and applications, Vol. 26, Siam, 1977.

[39] D. Carati, G. S. Winckelmans, H. Jeanmart, On the modelling of the subgrid-scale and filtered-scale stress tensors in large-eddy simulation, J Fluid Mech 441 (2001) 119-138.

[40] G. S. Winckelmans, A. A. Wray, O. V. Vasilyev, H. Jeanmart, Explicit-filtering large-eddy simulation using the tensor-diffusivity model supplemented by a dynamic smagorinsky term, Phys Fluids 13 (5) (2001) 1385-1403.

[41] M. de la Llave Plata, E. Lamballais, F. Naddei, On the performance of a high-order multiscale dg approach to les at increasing reynolds number, Computers Fluids 194 (2019) 104306.

[42] C. C. De Wiart, K. Hillewaert, L. Bricteux, G. Winckelmans, Implicit LES of free and wall-bounded turbulent flows based on the discontinuous Galerkin/symmetric interior penalty method, Int J Numer Meth Fluids 78 (6) (2015) $335-354$.

[43] G. Karamanos, G. E. Karniadakis, A spectral vanishing viscosity method for large-eddy simulations, J Comput Phys 163 (1) (2000) 22-50.

[44] J. Manzanero, E. Ferrer, G. Rubio, E. Valero, Design of a Smagorinsky Spectral Vanishing Viscosity turbulence model for discontinuous Galerkin methods, Comput Fluids (2020) 104440.

[45] A. A. Oberai, V. Gravemeier, B. G. C., Transfer of Energy in the variational multiscale formulation of LES, in: Proceedings of the 2004 Summer Program, 2004.

[46] F. Bassi, S. Rebay, A high-order accurate discontinuous finite element method for the numerical solution of the compressible navier-stokes equations, J Comput Phys 131 (2) (1997) 267-279.

[47] F. Bassi, A. Crivellini, S. Rebay, M. Savini, Discontinuous Galerkin solution of the Reynolds-averaged NavierStokes and k- $\omega$ turbulence model equations, Comput Fluids 34 (4-5) (2005) 507-540.

[48] D. Flad, G. Gassner, On the use of kinetic energy preserving DG-schemes for large eddy simulation, J Comput Phys 350 (2017) 782-795.

[49] S. Stolz, P. Schlatter, L. Kleiser, High-pass filtered eddy-viscosity models for large-eddy simulations of transitional and turbulent flow, Phys Fluids 17 (6) (2005) 065103.

[50] R. C. Moura, G. Mengaldo, J. Peiró, S. Sherwin, On the eddy-resolving capability of high-order discontinuous Galerkin approaches to implicit LES/under-resolved DNS of Euler turbulence, J Comput Phys 330 (2017) 615623.

[51] J. Meyers, P. Sagaut, B. J. Geurts, Optimal model parameters for multi-objective large-eddy simulations, Phys Fluids 18 (9) (2006) 095103

[52] F. Naddei, Adaptive large eddy simulations based on discontinuous galerkin methods, Ph.D. thesis, Paris Saclay (2019).

[53] F. Naddei, M. de la Llave Plata, E. Lamballais, V. Couaillier, M. Massot, M. Ihme, Large-scale space definition for the DG-VMS method based on energy transfer analyses, in: Proceedings of the 2018 Summer Program, 2018

[54] Z. Wang, A. Oberai, A mixed large eddy simulation model based on the residual-based variational multiscale formulation, Phys Fluids 22 (7) (2010) 075107.

[55] A. Leonard, A. Leonard, Large-eddy simulation of chaotic convection and beyond, in: 35th Aerospace Sciences Meeting and Exhibit, 1997, p. 204.

[56] J. Bardina, J. H. Ferziger, W. C. Reynolds, Improved turbulence models based on large eddy simulation of homogeneous, incompressible turbulent flows, Dept. Mech. Stanford Univ (1983).

[57] M. Frigo, S. G. Johnson, The design and implementation of FFTW3, Proceedings of the IEEE 93 (2) (2005) 216231, special issue on "Program Generation, Optimization, and Platform Adaptation".

[58] J.-Y. Lee, L. Greengard, The type 3 nonuniform FFT and its applications, J Comput Phys 206 (1) (2005) 1-5.

[59] G.-X. Fan, Q. H. Liu, Fast Fourier transform for discontinuous functions, IEEE T Antenn Propag 52 (2) (2004) $461-465$.

[60] C.-H. Zhu, Q. H. Liu, Y. Shen, L. Liu, A high accuracy conformal method for evaluating the discontinuous fourier transform, Prog Electromagn Res 109 (2010) 425-440. 